\title{
Forward $J / \psi$ and very backward jet inclusive production at the LHC
}

\author{
R. Boussarie* \\ Institute of Nuclear Physics, Polish Academy of Sciences, \\ Radzikowskiego 152, PL-31-342 Kraków, Poland \\ B. Ducloué ${ }^{\dagger}$ \\ Department of Physics, University of Jyväskylä, P.O. Box 35, 40014 University of Jyväskylä, Finland \\ and Helsinki Institute of Physics, P.O. Box 64, 00014 University of Helsinki, Finland \\ L. Szymanowski ${ }^{\ddagger}$ \\ National Center for Nuclear Research (NCBJ), 00-681 Warsaw, Poland \\ S. Wallon ${ }^{\S}$ \\ Laboratoire de Physique Théorique (UMR 8627), CNRS, Université Paris-Sud, \\ Université Paris-Saclay, 91405 Orsay Cedex, France \\ and UPMC, Université Paris 06, Faculté de Physique, 4 place Jussieu, 75252 Paris, France
}

(Received 19 October 2017; published 19 January 2018)

\begin{abstract}
In the spirit of Mueller-Navelet dijet production, we propose and study the inclusive production of a forward $J / \psi$ and a very backward jet at the LHC as an observable to reveal high-energy resummation effects à la Balitsky, Fadin, Kuraev, Lipatov. We obtain several predictions, which are based on the various mechanisms discussed in the literature to describe the production of the $J / \psi$, namely, nonrelativistic QCD singlet and octet contributions, and the color evaporation model.
\end{abstract}

DOI: $10.1103 /$ PhysRevD.97.014008

\section{INTRODUCTION}

The understanding of the high energy behavior of QCD in the perturbative Regge limit remains one of the most important and longstanding theoretical questions in particle physics. In the linear regime where gluonic saturation effects are not expected to be essential, QCD dynamics are described using the Balitsky, Fadin, Kuraev, Lipatov (BFKL) formalism [1-4], in the $k_{t}$-factorization [5-11] framework. In order to reveal these resummation effects, first with leading logarithmic (LL) precision, and more recently at next-toleading logarithmic (NLL) accuracy, many processes have been proposed. One of the most promising ones is the inclusive dijet production with a large rapidity separation, as proposed by Mueller and Navelet [12]. This idea led to many studies, now at the level of NLL precision.

\footnotetext{
*Renaud.Boussarie@ifj.edu.pl

†bertrand.b.ducloue@jyu.fi

${ }^{*}$ Lech.Szymanowski@ncbj.gov.pl

${ }^{\S}$ wallon@th.u-psud.fr
}

Published by the American Physical Society under the terms of the Creative Commons Attribution 4.0 International license. Further distribution of this work must maintain attribution to the author(s) and the published article's title, journal citation, and DOI. Funded by SCOAP ${ }^{3}$.
Recent $k_{t}$-factorization studies of Mueller-Navelet jets [13-20] were successful in describing such events at the LHC [21], exhibiting the very first sign of BFKL resummation effects at the LHC. To test the universality of such effects, we propose to apply a similar formalism to study the production of a forward $J / \psi$ meson and a very backward jet with a rapidity interval that is large enough to probe the BFKL dynamics but small enough for both the $J / \psi$ and the jet to be in the detector acceptance at LHC experiments such as ATLAS or CMS. ${ }^{1}$ Although $J / \psi$ mesons were first observed more than 40 years ago, the theoretical mechanism for their production is still to be fully understood and the validity of some models remains a subject of discussions (for recent reviews see for example Refs. [22-24]). In addition, most predictions for charmonium production rely on collinear factorization, in which one considers the interaction of two on-shell partons emitted by the incoming hadrons, to produce a charmonium accompanied by a fixed number of partons. On the contrary, in this work the $J / \psi$ meson and the tagged jet are produced by the

\footnotetext{
${ }^{1}$ For example, at CMS the CASTOR calorimeter allows one to tag a jet down to $Y_{2}=-6.55$ in rapidity while the $J / \psi$ could be reconstructed up to $Y_{1}=2.4$, thus with a maximum interval in rapidity of almost 9 , more than sufficient to see BFKL resummation effects.
} 
interaction of two collinear partons, but with the resummation of any number of accompanying unobserved partons, as usual in the $k_{t}$-factorization approach.

Here we will compare two different approaches for the description of charmonium production. First we will use the nonrelativistic QCD (NRQCD) formalism [25], in which the charmonium wave function is expanded as a series in powers of the relative velocity of its constituents. Next we will apply the color evaporation model (CEM), which relies on the local-duality hypothesis [26,27]. Finally we will show numerical estimates of the cross sections and of the azimuthal correlations between the $J / \psi$ and the jet obtained in both approaches. We will rely on the Brodsky-LepageMackenzie (BLM) procedure [28] to fix the renormalization scale, as it was adapted to the resummed perturbation theory à la BFKL in Refs. [29-32], which some of us applied to Mueller-Navelet jets in Ref. [15]. Below, we will only discuss in detail the new elements related to the various $J / \psi$ production mechanisms ${ }^{2}$ All details related to the BFKL evolution at NLL can be found in Refs. [13,14], while the details related to the application of the BLM scale fixing in our study are presented in Ref. [15].

\section{DETERMINATION OF THE $J / \psi$ MESON VERTEX}

We start with the determination of a general meson $M$ production vertex (the fact that we will restrict ourselves to $J / \psi$ in the rest of this paper plays no role at this stage). For the moment, we do not consider any specific model for its production. We generically denote with an index $M$ the kinematical variables attached to the system made of the meson and the possible accompanying unobserved particles, and use an index $V$ for the kinematical variables attached to the $J / \psi$ meson itself.

The inclusive high-energy hadroproduction process of such a meson $M$, via two gluon fusion, with a remnant $X$ and a jet with a remnant $Y$ separated by a large rapidity difference between the jet and the meson, in scattering of a hadron $H\left(p_{1}\right)$ with a hadron $H\left(p_{2}\right)$, is illustrated in Fig. 1, where as a matter of illustration, we consider the parton coming out of the hadron $H\left(p_{1}\right)$ to be a gluon and the parton coming out of the hadron $H\left(p_{2}\right)$ to be a quark. For the sake of illustration, we suppose that the meson is produced in the fragmentation region of the hadron $H\left(p_{1}\right)$, named as forward, while the jet is produced in the fragmentation region of the hadron $H\left(p_{2}\right)$, named as backward. On one hand, the longitudinal momentum fractions of the jet and of the meson are assumed to be large enough so that the usual collinear factorization applies (the hard scales are provided by the heavy meson mass and by the transverse momentum of the jet), and we can neglect any transverse momentum, denoting the momentum

\footnotetext{
${ }^{2}$ Note that in the context of $p A$ collisions, the computation of a similar vertex has been performed in Refs. [33,34], but based on a different factorization approach suitable for saturation studies.
}
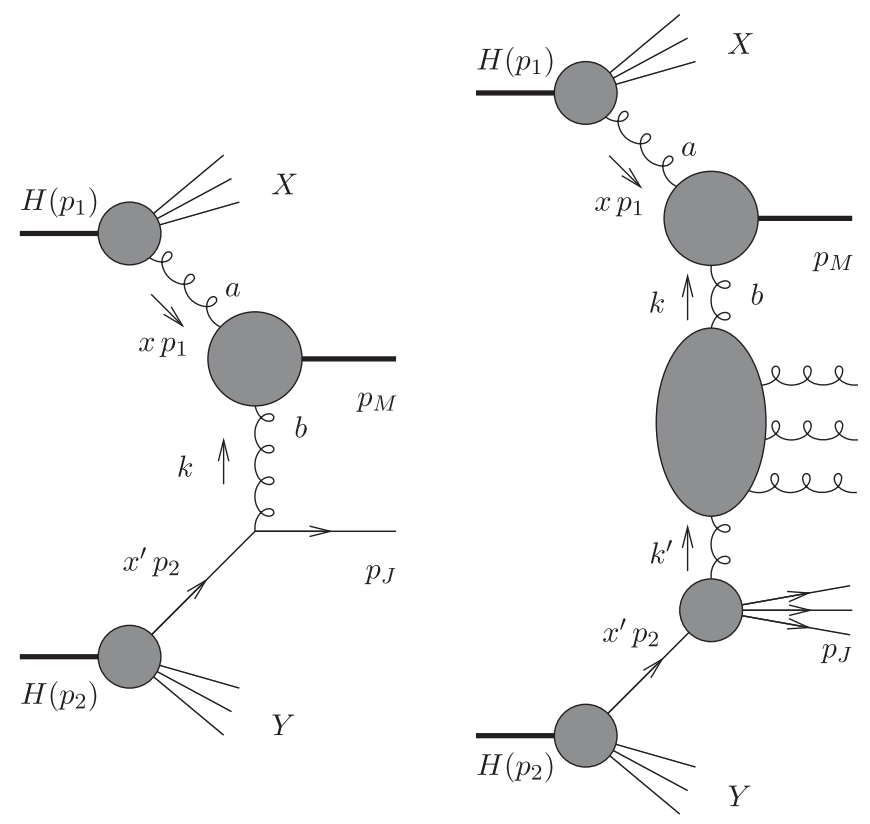

FIG. 1. The high-energy hadroproduction of a meson $M$ and a jet (here originating from a quark) with a large rapidity between them. Left: Born approximation. Right: inclusion of BFKL-like resummation effects due to multiple emissions of gluons and of higher order jet vertex corrections.

of the upper (resp. lower) parton as $x p_{1}$ (resp. $x^{\prime} p_{2}$ ), their distribution being given by usual parton distribution functions (PDFs). On the other hand, the $t$-channel exchanged momenta (e.g., $k$ in the lhs of Fig. 1, or the various ones involved in the rhs of Fig. 1) between the meson and the jet cannot be neglected due to their large relative rapidity, and we rely on $k_{t}$-factorization.

According to this picture, ${ }^{3}$ the differential cross section can be written as

$$
\begin{aligned}
& \frac{d \sigma}{d y_{V} d\left|p_{V \perp}\right| d \phi_{V} d y_{J} d\left|p_{J \perp}\right| d \phi_{J}} \\
& =\sum_{a, b} \int_{0}^{1} d x \int_{0}^{1} d x^{\prime} f_{a}(x) f_{b}\left(x^{\prime}\right) \\
& \quad \times \frac{d \hat{\sigma}}{d y_{V} d\left|p_{V \perp}\right| d \phi_{V} d y_{J} d\left|p_{J \perp}\right| d \phi_{J}},
\end{aligned}
$$

where $f_{a, b}$ are the standard parton distribution functions of a parton $a(b)$ in the according hadron.

In $k_{t}$-factorization, the partonic cross section reads

$$
\begin{aligned}
& \frac{d \hat{\sigma}}{d y_{V} d\left|p_{V \perp}\right| d \phi_{V} d y_{J} d\left|p_{J \perp}\right| d \phi_{J}} \\
& =\int d^{2} k_{\perp} d^{2} k_{\perp}^{\prime} V_{V, a}\left(k_{\perp}, x\right) G\left(-k_{\perp},-k_{\perp}^{\prime}, \hat{s}\right) V_{J, b}\left(-k_{\perp}^{\prime}, x^{\prime}\right),
\end{aligned}
$$

\footnotetext{
${ }^{3}$ We use the same notations as in Refs. $[13,14]$.
} 
where $G$ is the BFKL Green's function depending on $\hat{s}=x x^{\prime} s$, denoting as $\sqrt{s}$ the center-of-mass energy of the two colliding hadrons.

At leading order (LO), the jet vertex reads $[35,36]$ :

$$
\begin{gathered}
V_{J, a}^{(0)}\left(k_{\perp}, x\right)=h_{a}^{(0)}\left(k_{\perp}\right) \mathcal{S}_{J}^{(2)}\left(k_{\perp} ; x\right), \\
h_{a}^{(0)}\left(k_{\perp}\right)=\frac{\alpha_{s}}{\sqrt{2}} \frac{C_{A / F}}{k_{\perp}^{2}}, \\
\mathcal{S}_{J}^{(2)}\left(k_{\perp} ; x\right)=\delta\left(1-\frac{x_{J}}{x}\right)\left|p_{J \perp}\right| \delta^{(2)}\left(k_{\perp}-p_{J \perp}\right) .
\end{gathered}
$$

In the definition of $h_{\mathrm{a}}^{(0)}, C_{A}=N_{c}=3$ is to be used for an initial gluon and $C_{F}=\left(N_{c}^{2}-1\right) /\left(2 N_{c}\right)=4 / 3$ for an initial quark. Following the notations of Refs. [35,36], the dependence of $V$ on the jet variables is implicit. At nextto-leading order (NLO), the jet can be made of either a single or two partons. The explicit form of these jet vertices can be found in Ref. [13] as extracted from Refs. [35,36] after correcting a few misprints of Ref. [35].

The explicit form of the BFKL Green's function $G$, as obtained at LL [1-4] and at NLL [37,38] accuracy, can be found in Ref. [13], and will not be reproduced here.

In the rest of the present paper, we will only focus on the case where the meson vertex is treated at lowest order, while the Green's function and the jet vertex will be treated at NLL. The computation of the NLO $J / \psi$ vertex, which is a quite involved task, is left for further studies.

To properly fix the normalization, let us focus for a moment on the Born approximation, see the left-hand side (lhs) of Fig. 1. Then, each building block in the factorized formula (2.2) is treated at lowest order. In this limit, our normalizations are such that the Born Green's function is

$$
G^{\text {Born }}\left(k_{\perp}, k_{\perp}^{\prime}, \hat{s}\right)=\delta^{2}\left(k_{\perp}-k_{\perp}^{\prime}\right),
$$

while the jet vertices are given by Eqs. (2.3), (2.4). As explained above, the relevant components of the involved momenta read

$$
\begin{aligned}
k & =\beta p_{2}+k_{\perp}, \quad p_{J}=x^{\prime} p_{2}+p_{J \perp}, \\
p_{M} & =x p_{1}+p_{M \perp},
\end{aligned}
$$

where $k$ is the $t$-channel exchanged momentum. In the high-energy limit, the $\mathcal{T}_{M q}$-matrix reads

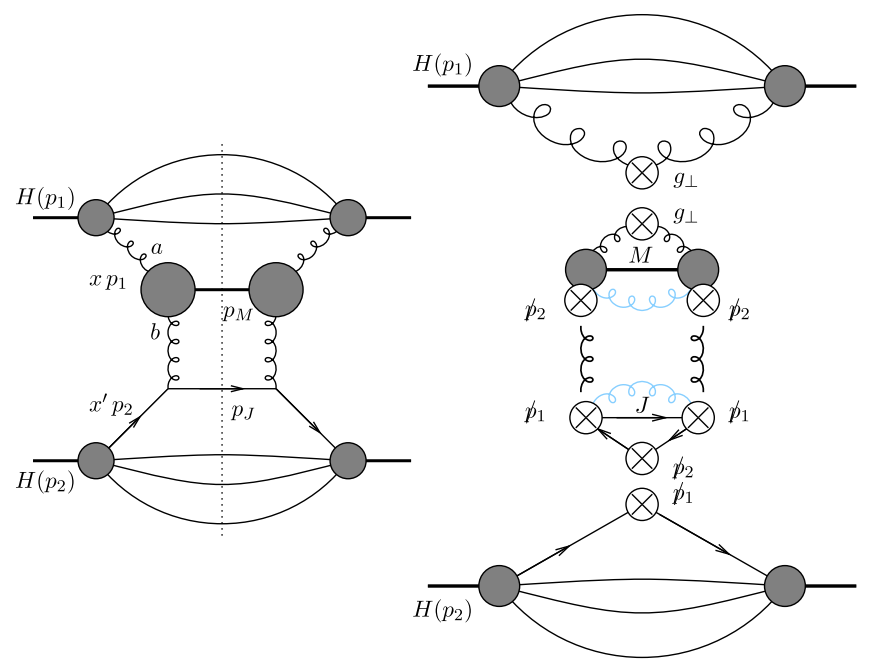

FIG. 2. Left: square of the amplitude of the Born process. Right: symbolic factorized form of this squared amplitude involving, from top to bottom, the gluonic PDF, the impact factor describing the $g g \rightarrow M$ transition, the $t$-channel exchange of two off-shell gluons (in bold), the vertex describing the $g q \rightarrow$ jet transition, and the quark PDF. The crosses symbolically denote the appropriate Fierz structure in Lorentz space. Namely, from top to bottom, $g_{\perp}$ tensors for gluons, due to the collinear factorization of the gluon out of the upper PDF, $\not \not_{1}$ and $\not \not_{2}$ arising from the nonsense polarizations of the $t$-channel gluons in $k_{t^{-}}$ factorization, and finally $\not \not_{2}$ and $\not p_{1}$ due to the collinear factorization of the quark out of the down PDF. The black fermions and curved gluon lines symbolize the trace over color and Lorentz indices after the use of the Fierz identity in these two spaces, while the blue (grey in printed black and white) gluons are traces over color after the use of the Fierz identity in color space.

$$
\begin{aligned}
\mathcal{T}_{M q}= & \frac{1}{i} \frac{2}{s} \frac{(-i)}{p_{J \perp}^{2}}\left\langle X\left|A_{\mu}^{a}(0)\right| H\left(p_{1}\right)\right\rangle \\
& \times g_{\perp}^{\mu \nu} \mathcal{A}_{\nu}^{a b} \bar{u}\left(p_{J}\right)\left(-i g \hat{p}_{1} t^{b}\right)\left\langle Y|q(0)| H\left(p_{2}\right)\right\rangle,
\end{aligned}
$$

where $a$ is the color index of a collinear gluon from the hadron $H\left(p_{1}\right)$ and $b$ is the color index of the exchanged $t$-channel gluon. Here $\mathcal{A}_{\nu}^{a b}$ denotes the $S$-matrix element describing the $g g \rightarrow M$ transition. Its computation will be discussed in detail in the following subsections. After factorization, illustrated symbolically by Fig. 2, we get

$$
\begin{aligned}
\sum_{M q} \mathcal{T}_{M q} \mathcal{T}_{M q}^{*}= & \frac{4}{s\left(p_{J \perp}^{2}\right)^{2}} \frac{1}{4 N\left(N^{2}-1\right)}\left\langle H\left(p_{1}\right)\left|A_{\mu^{\prime} \perp}^{a^{\prime}}(0)\right| X\right\rangle g_{\perp}^{\mu^{\prime} \nu^{\prime}}\left\langle X\left|A_{\nu^{\prime} \perp}^{a^{\prime}}(0)\right| H\left(p_{1}\right)\right\rangle \\
& \times \sum_{M} \mathcal{A}_{\mu \perp}^{a b} g_{\perp}^{\mu \nu}\left(\mathcal{A}_{\nu \perp}^{a b}\right)^{*} g^{2} \beta_{J}\left\langle H\left(p_{2}\right)\left|\bar{q}^{c}(0)\right| Y\right\rangle\left\langle Y\left|\hat{p}_{1} q^{c}(0)\right| H\left(p_{2}\right)\right\rangle .
\end{aligned}
$$

The phase space measure reads ${ }^{4}$

\footnotetext{
${ }^{4}$ This should be understood in an extended way, in particular due to the fact that $M$ might involve several particles, as it is the case for the color singlet NRQCD contribution.
} 


$$
d \Phi=(2 \pi)^{4} \delta\left(p_{1}+p_{2}-\left[p_{X}\right]-\left[p_{Y}\right]-\left[p_{M}\right]-p_{J}\right) \times\left[\frac{d^{3} p_{X}}{(2 \pi)^{3} 2 E_{X}}\right]\left[\frac{d^{3} p_{Y}}{(2 \pi)^{3} 2 E_{Y}}\right]\left[\frac{d^{3} p_{M}}{(2 \pi)^{3} 2 E_{M}}\right] \frac{d^{3} p_{J}}{(2 \pi)^{3} 2 E_{J}} .
$$

It can be written in a factorized form in terms of the rapidity $y_{J}$ of the quark jet and its transverse momentum $p_{J \perp}$ :

$$
\begin{aligned}
d \Phi= & \frac{2 \pi}{s} \int d^{2} k_{\perp} \delta^{2}\left(-\left[p_{M \perp}\right]+k_{\perp}\right) d x \delta\left(x-\left[\alpha_{M}\right]\right) \delta\left(1-x-\left[\alpha_{X}\right]\right)\left[\frac{d^{3} p_{X}}{(2 \pi)^{3} 2 E_{X}}\right]\left[\frac{d^{3} p_{M}}{(2 \pi)^{3} 2 E_{M}}\right] \\
& \times \delta^{2}\left(k_{\perp}+p_{J \perp}\right) d x^{\prime} \delta\left(x^{\prime}-\beta_{J}\right) \delta\left(1-x^{\prime}-\left[\beta_{Y}\right]\right)\left[\frac{d^{3} p_{Y}}{(2 \pi)^{3} 2 E_{Y}}\right] d y_{J} d^{2} p_{J \perp} .
\end{aligned}
$$

This $k_{t}$-factorization formula involves an integration over the transverse momentum $k_{t}$ of the four-momentum transfer $k$ in the $t$-channel between both vertices. Using the expressions of the unpolarized quark PDF

$$
H^{q}\left(x^{\prime}\right)=\frac{1}{s} \int\left[\frac{d^{3} p_{Y}}{(2 \pi)^{3} 2 E_{Y}}\right] \delta\left(1-x^{\prime}-\left[\beta_{Y}\right]\right)\left\langle H\left(p_{2}\right)|\bar{q}(0)| Y\right\rangle\left\langle Y\left|\hat{p}_{1} q(0)\right| H\left(p_{2}\right)\right\rangle,
$$

and of the unpolarized gluon PDF,

$$
\frac{g(x)}{x}=-\int\left[\frac{d^{3} p_{X}}{(2 \pi)^{3} 2 E_{X}}\right] \delta\left(1-x-\left[\alpha_{X}\right]\right)\left\langle H\left(p_{1}\right)\left|A_{\mu^{\prime} \perp}^{a^{\prime}}(0)\right| X\right\rangle g_{\perp}^{\mu^{\prime} \nu^{\prime}}\left\langle X\left|A_{\nu^{\prime} \perp}^{a^{\prime}}(0)\right| H\left(p_{1}\right)\right\rangle,
$$

we obtain an expression for the differential cross section

$$
\begin{aligned}
\frac{d \sigma}{d y_{J} d\left|p_{J \perp}\right| d \phi_{J}}= & \int d x g(x) d x^{\prime} H^{q}\left(x^{\prime}\right) d^{2} k_{\perp} \delta\left(x-\left[\alpha_{M}\right]\right) \delta^{2}\left(k_{\perp}-\left[p_{M \perp}\right]\right)\left[\frac{d^{3} p_{M}}{(2 \pi)^{3} 2 E_{M}}\right] \\
& \times \frac{8 \sqrt{2} \pi^{2}}{s^{2}\left(N^{2}-1\right)^{2} x k_{\perp}^{2}} \sum_{[M]} \mathcal{A}_{\mu \perp}^{a b} g_{\perp}^{\mu \nu}\left(\mathcal{A}_{\nu \perp}^{a b}\right)^{*} V_{J, q}^{(0)}\left(-k_{\perp}, x^{\prime}\right)
\end{aligned}
$$

in which we factorized out the vertex for quark jet production in the Born approximation,

$$
V_{J, q}^{(0)}\left(k_{\perp}, x^{\prime}\right)=\frac{g^{2}}{4 \pi \sqrt{2}} \frac{C_{F}}{\left|k_{\perp}\right|} \delta\left(1-\frac{x_{J}}{x^{\prime}}\right) \delta^{2}\left(k_{\perp}-p_{J \perp}\right),
$$

in accordance with Eqs. (2.3), (2.4).

\section{A. Color-singlet NRQCD contribution}

In the color-singlet contribution the system $[M]$ is made of the produced $J / \psi$ charmonium and of the unobserved gluon produced simultaneously with the charmonium in gluon-gluon fusion due to the negative charge-parity of the $J / \psi$. We parametrize the momentum $p_{V}$ of the $J / \psi$ and the momentum $l$ of the unobserved gluon in terms of Sudakov variables, as

$$
p_{V}=\alpha_{V} p_{1}+\frac{M_{J / \psi}^{2}-p_{V \perp}^{2}}{\alpha_{V} s} p_{2}+p_{V \perp}, \quad l=\alpha_{l} p_{1}-\frac{l_{\perp}^{2}}{\alpha_{l} s} p_{2}+l_{\perp}
$$

Thus the expression of

$$
\begin{aligned}
\delta\left(x-\left[\alpha_{M}\right]\right) \delta^{2}\left(k_{\perp}-\left[p_{M \perp}\right]\right)\left[\frac{d^{3} p_{M}}{(2 \pi)^{3} 2 E_{M}}\right] & =\delta\left(x-\alpha_{l}-\alpha_{V}\right) \delta^{2}\left(k_{\perp}-l_{\perp}-p_{V \perp}\right) \frac{d^{3} l}{(2 \pi)^{3} 2 E_{l}} \frac{d^{3} p_{V}}{(2 \pi)^{3} 2 E_{V}} \\
& =\frac{1}{4(2 \pi)^{6}} \delta\left(x-\alpha_{l}-\alpha_{V}\right) \delta^{2}\left(k_{\perp}-l_{\perp}-p_{V \perp}\right) \frac{d \alpha_{l} \theta\left(\alpha_{l}\right)}{\alpha_{l}} d^{2} l_{\perp} d y_{V} d^{2} p_{V \perp}
\end{aligned}
$$

permits, with the use of (2.13), to write the differential cross section in the form 
$\frac{d \sigma}{d y_{V} d\left|p_{V \perp}\right| d \phi_{V} d y_{J} d\left|p_{J \perp}\right| d \phi_{J}}=\int d x g(x) d y H^{q}(y) d^{2} k_{\perp} \frac{\left|p_{V \perp}\right| \sqrt{2}}{2^{5} \pi^{4} s^{2}\left(N^{2}-1\right)^{2} k_{\perp}^{2} x} \frac{\theta\left(x-\alpha_{V}\right)}{x-\alpha_{V}} \times \sum_{\lambda_{V}, \lambda_{l}} \mathcal{A}_{\mu \perp}^{a b} g_{\perp}^{\mu \nu}\left(\mathcal{A}_{\nu \perp}^{a b}\right)^{*} V_{q}^{(0)}\left(-k_{\perp}, y\right)$

from which we read off the $J / \psi$ production vertex of the color singlet NRQCD contribution as

$V_{J / \psi}^{(1)}=\frac{\left|p_{V \perp}\right| \sqrt{2}}{2^{5} \pi^{4} s^{2}\left(N^{2}-1\right)^{2} k_{\perp}^{2} x} \frac{\theta\left(x-\alpha_{V}\right)}{x-\alpha_{V}} \sum_{\lambda_{V}, \lambda_{l}} \mathcal{A}_{\mu \perp}^{a b} g_{\perp}^{\mu \nu}\left(\mathcal{A}_{\nu \perp}^{a b}\right)^{*}$.

One should note that the above expressions include an integration over the phase space of the unobserved gluon with momentum $l$. The vertex which allows to pass from open $q \bar{q}$ production to $J / \psi$ production in color singlet NRQCD reads $[39,40]$

$$
[v(q) \bar{u}(q)]_{\alpha \beta}^{i j} \rightarrow \frac{\delta^{i j}}{4 N}\left(\frac{\left\langle\mathcal{O}_{1}\right\rangle_{V}}{m}\right)^{1 / 2}\left[\hat{\epsilon}_{V}^{*}(2 \hat{q}+2 m)\right]_{\alpha \beta},
$$

with the momentum $q=\frac{1}{2} p_{V}, m$ being the mass of the charm quark, $M_{J / \psi}=2 m$. In the following we will use the nonperturbative coefficient $C_{1}$ defined as

$$
C_{1} \equiv\left(\frac{\left\langle\mathcal{O}_{1}\right\rangle_{V}}{m}\right)^{1 / 2}
$$

The matrix element $\left\langle O_{1}\right\rangle_{V}$ in NRQCD is related to the leptonic meson decay rate by [25]

$$
\Gamma\left[V \rightarrow l^{+} l^{-}\right]=\frac{2 e_{c}^{2} \pi \alpha^{2}}{3} \frac{\left\langle\mathcal{O}_{1}\right\rangle_{V}}{m^{2}}\left(1-\frac{16 \alpha_{s}}{3 \pi}\right) .
$$

Here $\alpha$ is the fine-structure constant and $e_{c}=2 / 3$ is the electric charge of the charm quark. Equation (2.21) includes the one-loop QCD correction [41-43] and $\alpha_{s}$ is the strong coupling constant. One can use the value of this decay rate to fix $\left\langle\mathcal{O}_{1}\right\rangle_{V}$ through this relation. Namely, using the values $\Gamma_{e^{+} e^{-}}=5.55 \times 10^{-6} \mathrm{GeV}$ [44], $m=1.5 \mathrm{GeV}$ and a three-loop running coupling with $\Lambda_{4}=0.305 \mathrm{GeV}$, we obtain $\left\langle\mathcal{O}_{1}\right\rangle_{J / \psi}=0.444 \mathrm{GeV}^{3}$. As quoted in Ref. [45], recent phenomenological analyses [46-48] have used slightly smaller values of either 0.387 or $0.440 \mathrm{GeV}^{3}$, as obtained in Refs. [49,50] respectively. In order not to underestimate the uncertainty, in the following we will vary $\left\langle\mathcal{O}_{1}\right\rangle_{J / \psi}$ between 0.387 and $0.444 \mathrm{GeV}^{3}$.

The momentum transfer $k$ in the $t$-channel entering the charmonium vertex has the approximate form given by Eq. (2.6). The momentum conservation in the charmonium vertex $x p_{1}+k=p_{V}+l$ leads to the following relations between the Sudakov variables of momenta:

$$
\begin{aligned}
& x=\alpha_{V}+\alpha_{l}, \quad k_{\perp}=p_{V \perp}+l_{\perp}, \\
& \beta=\frac{4 m^{2}-p_{V \perp}^{2}}{\alpha_{V} s}-\frac{l_{\perp}^{2}}{\alpha_{l} s} .
\end{aligned}
$$

The contribution to the hard part is given by the 6 diagrams shown in Fig. 3, which leads to the expressions

$$
\begin{gathered}
D_{1}=\frac{(-i g)^{3} i^{2} C_{1}}{4 N} \operatorname{tr}_{c}\left(t^{l} t^{a} t^{b}\right) \operatorname{Tr}\left[\hat{\epsilon}_{V}^{*}(2 \hat{q}+2 m) \hat{\epsilon}^{*}(l) \frac{\hat{q}+\hat{l}+m}{(q+l)^{2}-m^{2}} \gamma_{\perp}^{\mu} \frac{\beta \hat{p}_{2}+\hat{k}_{\perp}-\hat{q}+m}{\left(\beta p_{2}+k_{\perp}-q\right)^{2}-m^{2}} \hat{p}_{2}\right], \\
D_{2}=\frac{(-i g)^{3} i^{2} C_{1}}{4 N} \operatorname{tr}_{c}\left(t^{a} t^{l} t^{b}\right) \operatorname{Tr}\left[\hat{\epsilon}_{V}^{*}(2 \hat{q}+2 m) \gamma_{\perp}^{\mu} \frac{\hat{q}-x \hat{p}_{1}+m}{\left(q-x p_{1}\right)^{2}-m^{2}} \hat{\epsilon}^{*}(l) \frac{\beta \hat{p}_{2}+\hat{k}_{\perp}-\hat{q}+m}{\left(\beta p_{2}+k_{\perp}-q\right)^{2}-m^{2}} \hat{p}_{2}\right], \\
D_{3}=\frac{(-i g)^{3} i^{2} C_{1}}{4 N} \operatorname{tr}_{c}\left(t^{a} t^{b} t^{l}\right) \operatorname{Tr}\left[\hat{\epsilon}_{V}^{*}(2 \hat{q}+2 m) \gamma_{\perp}^{\mu} \frac{\hat{q}-x \hat{p}_{1}+m}{\left(q-x p_{1}\right)^{2}-m^{2}} \hat{p}_{2} \frac{-\hat{q}-\hat{l}+m}{(-q-l)^{2}-m^{2}} \hat{\epsilon}^{*}(l)\right], \\
D_{4}=\frac{(-i g)^{3} i^{2} C_{1}}{4 N} \operatorname{tr}_{c}\left(t^{l} t^{b} t^{a}\right) \operatorname{Tr}\left[\hat{\epsilon}_{V}^{*}(2 \hat{q}+2 m) \hat{\epsilon}^{*}(l) \frac{\hat{q}+\hat{l}+m}{(q+l)^{2}-m^{2}} \hat{p}_{2} \frac{x \hat{p}_{1}-\hat{q}+m}{\left(x p_{1}-q\right)^{2}-m^{2}} \gamma_{\perp}^{\mu}\right], \\
D_{5}=\frac{(-i g)^{3} i^{2} C_{1}}{4 N} \operatorname{tr}_{c}\left(t^{b} t^{l} t^{a}\right) \operatorname{Tr}\left[\hat{\epsilon}_{V}^{*}(2 \hat{q}+2 m) \hat{p}_{2} \frac{\hat{q}-\beta \hat{p}_{2}-\hat{k}_{\perp}+m}{\left(q-\beta p_{2}-k_{\perp}\right)^{2}-m^{2}} \hat{\epsilon}^{*}(l) \frac{x \hat{p}_{1}-\hat{q}+m}{\left(x p_{1}-q\right)^{2}-m^{2}} \gamma_{\perp}^{\mu}\right],
\end{gathered}
$$




$$
D_{6}=\frac{(-i g)^{3} i^{2} C_{1}}{4 N} \operatorname{tr}_{c}\left(t^{b} t^{a} t^{l}\right) \operatorname{Tr}\left[\hat{\epsilon}_{V}^{*}(2 \hat{q}+2 m) \hat{p}_{2} \frac{\hat{q}-\beta \hat{p}_{2}-\hat{k}_{\perp}+m}{\left(q-\beta p_{2}-k_{\perp}\right)^{2}-m^{2}} \gamma_{\perp}^{\mu} \frac{-\hat{q}-\hat{l}+m}{(-q-l)^{2}-m^{2}} \hat{\epsilon}^{*}(l)\right],
$$

where $\operatorname{tr}_{c}$ and $\operatorname{Tr}$ denote respectively the color and the Dirac traces. Let us observe the following relations between the Dirac traces $\operatorname{Tr}_{D(i)}$ of diagrams $D(i)$ due to charge conjugation invariance:

$$
\operatorname{Tr}_{D(1)}=\operatorname{Tr}_{D(6)}, \quad \operatorname{Tr}_{D(2)}=\operatorname{Tr}_{D(5)}, \quad \operatorname{Tr}_{D(3)}=\operatorname{Tr}_{D(4)} .
$$

Consider the color factor: the symmetry property (2.29) results in the appearance in the sum of all diagrams of the symmetric structure constants $d^{c k l}$ of the $S U(N)$ color group only. Thus, we obtain

$$
\begin{aligned}
\mathcal{A}_{\mu \perp}^{a b}= & \sum_{i=1}^{6} D(i)=\frac{(-i g)^{3} i^{2} C_{1}}{4 N} \frac{d^{a b l}}{4}\left\{\frac{2 \operatorname{Tr}_{D(1) \mu \perp}}{\left[(q+l)^{2}-m^{2}\right]\left[\left(\beta p_{2}+k_{\perp}-q\right)^{2}-m^{2}\right]}\right. \\
& \left.+\frac{2 \operatorname{Tr}_{D(2) \mu \perp}}{\left[\left(q-x p_{1}\right)^{2}-m^{2}\right]\left[\left(\beta p_{2}+k_{\perp}-q\right)^{2}-m^{2}\right]}+\frac{2 \operatorname{Tr}_{D(3) \mu \perp}}{\left[\left(q-x p_{1}\right)^{2}-m^{2}\right]\left[(l+q)^{2}-m^{2}\right]}\right\} \\
\equiv & \frac{(-i g)^{3} i^{2} C_{1}}{4 N} \frac{d^{a b l}}{4} \mathcal{D}_{\mu \perp}^{\nu \rho} \epsilon_{V \rho}^{*}(2 q) \epsilon_{\nu}^{*}(l),
\end{aligned}
$$

where we introduced the shorthand notation $\mathcal{D}_{\mu \perp}^{\nu \rho} \epsilon_{V \rho}^{*}(2 q) \epsilon_{\nu}^{*}(l)$ for the sum of all six diagrams contributing to $J / \psi$ production within the color singlet mechanism. One can check that this sum vanishes in the limit $k_{\perp} \rightarrow 0$, as it should be the case for an impact factor in $k_{t}$-factorization due to its gauge invariance. For the gluon $g(l)$, we choose the gauge ${ }^{5}$

$$
p_{2} \cdot \epsilon^{*}(l)=0,
$$

which is a natural choice for a meson emitted in the fragmentation region of the hadron of momentum $p_{1}$. The three different traces then read

$$
\begin{aligned}
\operatorname{Tr}_{D(1)}^{\mu \perp}= & 2 m \operatorname{Tr}\left[m^{2} \hat{\epsilon}_{V}^{*} \hat{\epsilon}^{*}(l) \gamma_{\perp}^{\mu} \hat{p}_{2}+\hat{\epsilon}_{V}^{*} \hat{\epsilon}^{*}(l)(\hat{q}+\hat{l}) \gamma_{\perp}^{\mu}\left(\hat{k}_{\perp}-\hat{q}\right) \hat{p}_{2}+\hat{\epsilon}_{V}^{*} \hat{q} \hat{\epsilon}^{*}(l)(\hat{q}+\hat{l}) \gamma_{\perp}^{\mu} \hat{p}_{2}+\hat{\epsilon}_{V}^{*} \hat{q} \hat{\epsilon}^{*}(l) \gamma_{\perp}^{\mu}\left(\hat{k}_{\perp}-\hat{q}\right) \hat{p}_{2}\right] \\
= & 8 m\left[k_{\perp}^{\mu}\left(2 \epsilon^{*}(l) \cdot q \epsilon_{V}^{*} \cdot p_{2}+\epsilon_{V}^{*} \cdot \epsilon^{*}(l) p_{2} \cdot l\right)-\epsilon_{V \perp}^{* \mu}\left(k_{\perp} \cdot \epsilon^{*}(l) p_{2} \cdot l+4 p_{2} \cdot q q \cdot \epsilon^{*}(l)\right)\right. \\
& \left.+l_{\perp}^{\mu}\left(p_{2} \cdot \epsilon_{V}^{*} k_{\perp} \cdot \epsilon^{*}(l)-2 p_{2} \cdot q \epsilon_{V}^{*} \cdot \epsilon^{*}(l)\right)+\epsilon_{\perp}^{* \mu}(l)\left(-k_{\perp} \cdot l_{\perp} p_{2} \cdot \epsilon_{V}^{*}+k_{\perp} \cdot \epsilon_{V}^{*} p_{2} \cdot l+2 p_{2} \cdot q \epsilon_{V}^{*} \cdot l\right)\right], \\
\operatorname{Tr}_{D(2)}^{\mu \perp}= & 2 m \operatorname{Tr}\left[m^{2} \gamma_{\perp}^{\mu} \hat{\epsilon}^{*}(l) \hat{p}_{2} \hat{\epsilon}_{V}^{*}+\hat{\epsilon}_{V}^{*} \hat{q} \gamma_{\perp}^{\mu} \hat{\epsilon}^{*}(l)\left(\hat{k}_{\perp}-\hat{q}\right) \hat{p}_{2}+\hat{\epsilon}_{V}^{*} \hat{q} \gamma_{\perp}^{\mu}\left(\hat{q}-x \hat{p}_{1}\right) \hat{\epsilon}^{*}(l) \hat{p}_{2}+\hat{\epsilon}_{V}^{*} \gamma_{\perp}^{\mu}\left(\hat{q}-x \hat{p}_{1}\right) \hat{\epsilon}^{*}(l)\left(\hat{k}_{\perp}-\hat{q}\right) \hat{p}_{2}\right] \\
= & 2 m\left\{8 q_{\perp}^{\mu}\left(-2 p_{2} \cdot q \epsilon_{V}^{*} \cdot \epsilon^{*}(l)+\epsilon^{*}(l) \cdot k_{\perp} p_{2} \cdot \epsilon_{V}^{*}\right)+8 x p_{2} \cdot q\left(\epsilon_{V \perp}^{* \mu} p_{1} \cdot \epsilon^{*}(l)-\epsilon_{\perp}^{* \mu}(l) p_{1} \cdot \epsilon_{V}^{*}\right)\right. \\
& \left.+x\left[-2 s \epsilon_{V \perp}^{* \mu} k_{\perp} \cdot \epsilon^{*}(l)-2 s \epsilon_{\perp}^{* \mu}(l) k_{\perp} \cdot \epsilon_{V}^{*}+k_{\perp}^{\mu}\left(2 s \epsilon_{V}^{*} \cdot \epsilon^{*}(l)-4 p_{2} \cdot \epsilon_{V}^{*} p_{1} \cdot \epsilon^{*}(l)\right)\right]\right\}
\end{aligned}
$$

and

$$
\begin{aligned}
\operatorname{Tr}_{D(3)}^{\mu \perp}= & 2 m \operatorname{Tr}\left[m^{2} \hat{\epsilon}_{V}^{*} \gamma_{\perp}^{\mu} \hat{p}_{2} \hat{\epsilon}^{*}(l)-\hat{\epsilon}_{V}^{*} \gamma_{\perp}^{\mu}\left(\hat{q}-x \hat{p}_{1}\right) \hat{p}_{2}(\hat{q}+\hat{l}) \hat{\epsilon}^{*}(l)-\hat{\epsilon}_{V}^{*} \hat{q} \gamma_{\perp}^{\mu} \hat{p}_{2}(\hat{q}+\hat{l}) \hat{\epsilon}^{*}(l)+\hat{\epsilon}_{V}^{*} \hat{q} \gamma_{\perp}^{\mu}\left(\hat{q}-x \hat{p}_{1}\right) \hat{p}_{2} \hat{\epsilon}^{*}(l)\right] \\
= & 2 m\left\{8 q_{\perp}^{\mu}\left(-2 q \cdot \epsilon^{*}(l) p_{2} \cdot \epsilon_{V}^{*}+\epsilon_{V}^{*} \cdot l p_{2} \cdot \epsilon^{*}(l)-p_{2} \cdot l \epsilon_{V}^{*} \cdot \epsilon^{*}(l)\right)+x\left[4 s \epsilon_{V \perp}^{* \mu} q \cdot \epsilon^{*}(l)\right.\right. \\
& \left.\left.+2 s\left(-\epsilon_{\perp}^{* \mu}(l) l \cdot \epsilon_{V}^{*}+l_{\perp}^{\mu} \epsilon^{*}(l) \cdot \epsilon_{V}^{*}\right)-4 l_{\perp}^{\mu} p_{1} \cdot \epsilon^{*}(l) p_{2} \cdot \epsilon_{V}^{*}-4 \epsilon_{\perp}^{* \mu}(l)\left(p_{1} \cdot \epsilon_{V}^{*} p_{2} \cdot l-p_{1} \cdot l p_{2} \cdot \epsilon_{V}^{*}\right)+4 \epsilon_{V \perp}^{* \mu} p_{2} \cdot l p_{1} \cdot \epsilon^{*}(l)\right]\right\}
\end{aligned}
$$

The denominators appearing in the expression for $\mathcal{A}_{\mu \perp}^{a b}$ are equal to

$$
\begin{aligned}
(q+l)^{2}-m^{2} & =\frac{1}{2}\left[k_{\perp}^{2}+4 m^{2}\left(\frac{x}{\alpha_{V}}-1\right)-\frac{x}{\alpha_{V}} p_{V \perp}^{2}-\frac{x}{\alpha_{l}} l_{\perp}^{2}\right], \\
\left(q-x p_{1}\right)^{2}-m^{2} & =-\frac{x}{2 \alpha_{V}}\left(4 m^{2}-p_{V \perp}^{2}\right),\left(\beta p_{2}+k_{\perp}-q\right)^{2}-m^{2}=\frac{1}{2}\left(k_{\perp}^{2}-4 m^{2}+\frac{x}{\alpha_{l}} l_{\perp}^{2}\right) .
\end{aligned}
$$

\footnotetext{
${ }^{5}$ Note that the sum of diagrams in this color singlet mechanism is gauge invariant, although the $t$-channel gluon is off-shell: indeed due to the simple single color structure $d^{a b l}$ which factorizes, they are QED like.
} 


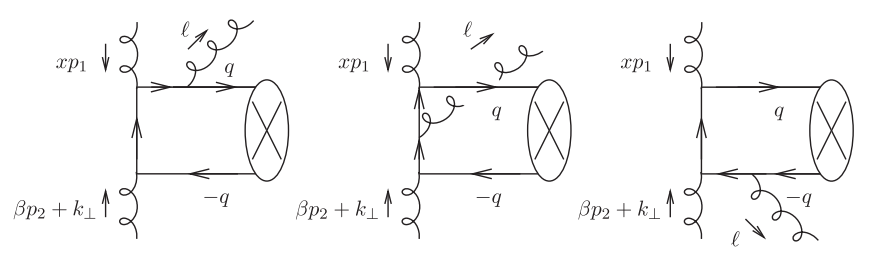

$D_{1}$

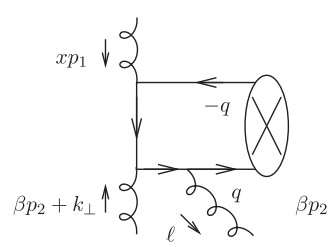

$D_{4}$
$D_{2}$

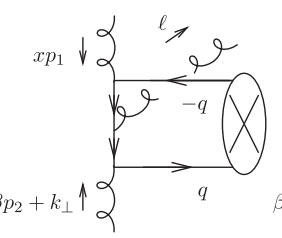

$D_{5}$
$D_{3}$

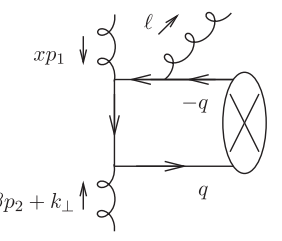

$D_{6}$

FIG. 3. The 6 diagrams contributing to the amplitude in color singlet NRQCD. The blobs with a cross symbolize the Fierz structure of Eq. (2.19).

The cross section is obtained by squaring the sum of diagrams $D(i)$, i.e. by contracting this sum with its complex conjugate through the polarization tensors for the $J / \psi$ and

the gluon $g(l)$ and the projection operator related to the factorization of the gluonic PDF, namely

$$
\begin{aligned}
\mathcal{D}^{(1)}(J / \psi) \equiv & \mathcal{D}^{\mu \nu \rho} g_{\perp \mu \mu^{\prime}}\left(-g_{\rho \rho^{\prime}}+\frac{q_{\rho} q_{\rho^{\prime}}}{m^{2}}\right) \\
& \times\left(-g_{\nu \nu^{\prime}}+\frac{p_{2 \nu} l_{\nu^{\prime}}+p_{2 \nu^{\prime}} l_{\nu}}{p_{2} \cdot l}\right) \mathcal{D}^{* \mu^{\prime} \nu^{\prime} \rho^{\prime}} .
\end{aligned}
$$

Thus we obtain that

$\sum_{\lambda_{V} \lambda_{l}} \mathcal{A}_{\mu \perp}^{a b} g_{\perp}^{\mu \nu}\left(\mathcal{A}_{\nu \perp}^{a b}\right)^{*}=\frac{g^{6} C_{1}^{2}}{(4 N)^{2}} \frac{d^{a b l} d^{a b l}}{4^{2}} \mathcal{D}^{(1)}(J / \psi)$,

which by taking into account Eq. (2.18) gives the $J / \psi$ production vertex in the form

$V_{J / \psi}^{(1)}=\frac{\left|p_{V \perp}\right| \sqrt{2} g^{6} C_{1}^{2}}{s^{2} \pi^{4} 2^{13} k_{\perp}^{2}} \frac{d^{a b l} d^{a b l}}{N^{2}\left(N^{2}-1\right)^{2}} \frac{\theta\left(x-\alpha_{V}\right)}{x\left(x-\alpha_{V}\right)} \mathcal{D}^{(1)}(J / \psi)$,

with $\alpha_{V}=\frac{\sqrt{4 m^{2}-p_{V \perp}^{2}}}{\sqrt{s}} e^{y_{V}}$ and $d^{a b l} d^{a b l}=\frac{\left(N^{2}-4\right)\left(N^{2}-1\right)}{N}$. The final expression for $\mathcal{D}^{(1)}(J / \psi)$ reads

$$
\begin{aligned}
\mathcal{D}^{(1)}(J / \psi)= & \frac{2^{9}}{\left(m^{2}-q_{\perp}^{2}\right)^{2}\left(4 x k_{\perp} \cdot q_{\perp}+k_{\perp}^{2}\left(\alpha_{V}-2 x\right)+4 m^{2}\left(x-\alpha_{V}\right)-4 x q_{\perp}^{2}\right)^{2}} \\
& \times \frac{s^{2} \alpha_{V}^{2}\left(\alpha_{V}-x\right)^{2}}{\left(4 x\left(x q_{\perp}^{2}-\alpha_{V} k_{\perp} \cdot q_{\perp}\right)+k_{\perp}^{2} \alpha_{V}^{2}-4 m^{2}\left(\alpha_{V}-x\right)^{2}\right)^{2}}\left\{32 m^{4} \alpha_{V}^{2}\left(\alpha_{V}-x\right)^{2}\left(k_{\perp} \cdot q_{\perp}\right)^{2}\right. \\
& +\left(k_{\perp}^{2}\right)^{3} \alpha_{V}^{2}\left[m^{2}\left(\alpha_{V}^{2}-2 x \alpha_{V}+2 x^{2}\right)-q_{\perp}^{2}\left(\alpha_{V}-x\right)^{2}\right] \\
& +8 m^{2} k_{\perp}^{2}\left[-2 \alpha_{V} k_{\perp} \cdot q_{\perp}\left(m^{2}\left(\alpha_{V}-x\right)^{3}+q_{\perp}^{2}\left(2 \alpha_{V}^{3}+2 x^{2} \alpha_{V}-3 x \alpha_{V}^{2}+x^{3}\right)\right)+\alpha_{V}^{2}\left(\alpha_{V}^{2}-2 x \alpha_{V}+3 x^{2}\right)\left(k_{\perp} \cdot q_{\perp}\right)^{2}\right. \\
& \left.+2\left(m^{2}\left(\alpha_{V}-x\right)^{2}-q_{\perp}^{2}\left(\alpha_{V}^{2}-x \alpha_{V}+x^{2}\right)\right)^{2}\right]-4\left(k_{\perp}^{2}\right)^{2}\left[\alpha_{V} k_{\perp} \cdot q_{\perp}\left(m^{2}\left(\alpha_{V}^{3}+x^{2} \alpha_{V}-x \alpha_{V}^{2}+x^{3}\right)-x q_{\perp}^{2}\left(\alpha_{V}-x\right)^{2}\right)\right. \\
& \left.\left.+m^{4}\left(\alpha_{V}-x\right)^{4}+m^{2} q_{\perp}^{2}\left(-5 \alpha_{V}^{4}+6 x^{3} \alpha_{V}-13 x^{2} \alpha_{V}^{2}+12 x \alpha_{V}^{3}-2 x^{4}\right)+x^{2}\left(q_{\perp}^{2}\right)^{2}\left(\alpha_{V}-x\right)^{2}\right]\right\} .
\end{aligned}
$$

\section{B. Color-octet NRQCD contribution}

In the color-octet contribution $[M]$ denotes one meson state, thus

$$
\begin{gathered}
\delta\left(x-\left[\alpha_{M}\right]\right) \delta^{2}\left(k_{\perp}-\left[p_{M \perp}\right]\right)\left[\frac{d^{3} p_{M}}{(2 \pi)^{3} 2 E_{M}}\right] \\
=\frac{\delta\left(x-\alpha_{V}\right) \delta^{2}\left(k_{\perp}-p_{V \perp}\right)}{2(2 \pi)^{3}} d y_{V} d^{2} p_{V \perp},
\end{gathered}
$$

which leads to the differential cross section

$$
\begin{aligned}
& \frac{d \sigma}{d y_{V} d\left|p_{V \perp}\right| d \phi_{V} d y_{J} d\left|p_{J \perp}\right| d \phi_{J}} \\
& =\int d x g(x) d y H^{q}(y) d^{2} k_{\perp} \frac{\left|p_{V \perp}\right| \delta\left(x-\alpha_{V}\right) \delta^{2}\left(k_{\perp}-p_{V \perp}\right)}{\sqrt{2} \pi s^{2}\left(N^{2}-1\right)^{2} k_{\perp}^{2} x} \\
& \quad \times \sum_{\lambda_{V}} \mathcal{A}_{\mu \perp}^{a b} g_{\perp}^{\mu \nu}\left(\mathcal{A}_{\nu \perp}^{a b}\right)^{*} V_{q}^{(0)}\left(-k_{\perp}, y\right),
\end{aligned}
$$

from which we read off the $J / \psi$ production vertex of the color octet NRQCD contribution:

$$
\begin{aligned}
V_{J / \psi}^{(8)}\left(k_{\perp}, x\right)= & \frac{\left|p_{V \perp}\right| \delta\left(x-\alpha_{V}\right) \delta^{2}\left(k_{\perp}-p_{V \perp}\right)}{\sqrt{2} \pi s^{2}\left(N^{2}-1\right)^{2} k_{\perp}^{2} x} \\
& \times \sum_{\lambda_{V}} \mathcal{A}_{\mu \perp}^{a b} g_{\perp}^{\mu \nu}\left(\mathcal{A}_{\nu \perp}^{a b}\right)^{*} .
\end{aligned}
$$

The vertex which allows to pass from open $q \bar{q}$ production to $J / \psi$ production in color octet NRQCD is defined as

$$
[v(q) \bar{u}(q)]_{\alpha \beta}^{i j \rightarrow d} \rightarrow t_{i j}^{d} d_{8}\left(\frac{\left\langle\mathcal{O}_{8}\right\rangle_{V}}{m}\right)^{1 / 2}\left[\hat{\epsilon}_{V}^{*}(2 \hat{q}+2 m)\right]_{\alpha \beta},
$$

where the value of the coefficient $d_{8}$ is determined by comparison with the result of Cho and Leibovich [51,52], 


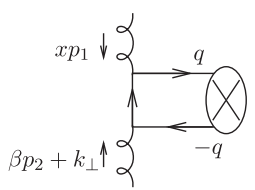

A

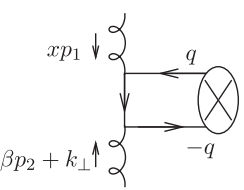

B

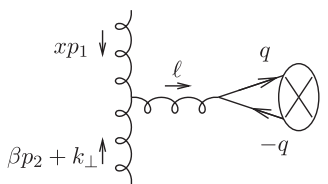

C
FIG. 4. The 3 diagrams contributing to the amplitude in color octet NRQCD. The blobs with a cross symbolize the Fierz structure of Eq. (2.43).

namely Eq. (A.1b) of Ref. [52], for the total squared amplitude for creating a specific quarkonium state ${ }^{3} S_{1}^{(8)}$. Note that here we only consider the case where the quarkantiquark pair has the same spin and orbital momentum as the $J / \psi$ meson. At large transverse momentum, which is the case we will consider in the following, this contribution is found to be dominant, see e.g., Ref. [53]. For $N=3$ the coefficient $d_{8}$ equals $d_{8}=\frac{1}{4 \sqrt{3}}$. An early analysis [54] gave for the nonperturbative coefficient

$$
C_{8} \equiv\left(\frac{\left\langle\mathcal{O}_{8}\right\rangle_{V}}{m}\right)^{1 / 2}
$$

values between $3.2 \times 10^{-4}$ and $5 \times 10^{-4} \mathrm{GeV}^{3}$. More recent analyses [46-48], as quoted in Ref. [45], obtained significantly larger values which we will use here, namely we will vary $\left\langle\mathcal{O}_{8}\right\rangle_{J / \psi}$ between $0.224 \times 10^{-2}$ and $1.1 \times 10^{-2} \mathrm{GeV}^{3}$.

The hard part corresponds to the sum of the three diagrams of Fig. 4, namely

$$
\begin{aligned}
\mathcal{A}_{\perp}^{a b \mu}(A+B+C)= & (-i g)^{2} i \bar{u}(q)\left[t^{a} t^{b} \gamma_{\perp}^{\mu} \frac{\hat{q}-x \hat{p}_{1}+m}{\left(q-x p_{1}\right)^{2}-m^{2}} \hat{p}_{2}\right. \\
& +t^{b} t^{a} \hat{p}_{2} \frac{x \hat{p}_{1}-\hat{q}+m}{\left(x p_{1}-q\right)^{2}-m^{2}} \gamma_{\perp}^{\mu} \\
& \left.-i f^{a b c}\left(-2 k_{\perp}^{\mu} p_{2}^{\rho}+4 p_{2} \cdot q g_{\perp}^{\mu \rho}\right) \frac{t^{c} \gamma_{\rho}}{4 q^{2}}\right] v(q) .
\end{aligned}
$$

After taking into account the projection (2.43) we obtain

$$
\begin{aligned}
& \mathcal{A}_{\perp}^{a b \mu}(A+B+C \rightarrow J / \psi)_{8} \\
& =(-i g)^{2} i^{2} f^{a b d} \frac{1}{2}\left\{\frac{8 m}{\left(q-x p_{1}\right)^{2}-m^{2}}\left[-2 q \cdot p_{2} \epsilon_{V \perp}^{* \mu}+k_{\perp}^{\mu} p_{2} \cdot \epsilon_{V}^{*}\right]\right. \\
& \left.-\frac{16 m}{4 q^{2}}\left[-k_{\perp}^{\mu} p_{2} \cdot \epsilon_{\perp}^{*}+2 p_{2} \cdot q \epsilon_{V \perp}^{* \mu}\right]\right\} d_{8} \mathcal{C}_{8}
\end{aligned}
$$

in which the propagator $\left(q-x p_{1}\right)^{2}-m^{2}=-\frac{1}{2}\left(4 m^{2}-k_{\perp}^{2}\right)$. One can easily check that this sum vanishes in the limit $k_{\perp} \rightarrow 0$, as it should be the case for an impact factor in $k_{t^{-}}$ factorization. This is also true at the level of open quark production, see Eq. (2.45). The result (2.46) together with Eq. (2.42) leads to the $J / \psi$ production vertex for $N=3$ :

$$
\begin{aligned}
V_{J / \psi}^{(8)}= & -\delta\left(x-\alpha_{V}\right) \delta^{2}\left(k_{\perp}-p_{V \perp}\right) \\
& \times \frac{\left|p_{V \perp}\right| \sqrt{2} g^{4} k_{\perp}^{2} x}{128 \pi m^{3}\left(4 m^{2}-k_{\perp}^{2}\right)^{2}}\left\langle\mathcal{O}_{8}\right\rangle_{V} .
\end{aligned}
$$

\section{Color evaporation model}

In the color evaporation model $[M]$ denotes an open quark-antiquark produced state with an invariant mass $M$. Moreover, the differential cross section in this model involves an integration over the invariant mass $M^{2}$ in the interval $\left[4 m^{2}, 4 M_{D}^{2}\right]$, as it is assumed that in this interval below the $D$-meson mass threshold, a fixed fraction of these $c \bar{c}$ pairs (either produced in a singlet or in an octet color state) will form $J / \psi$ bound states. This fraction is parametrized by the constant $F_{J / \psi}$, which is assumed to be universal as one of the main assumptions of the color evaporation model, and we will vary it between 0.02 and 0.04 based on a recent analysis [55] (see also Ref. [56]).

The $J / \psi$ momentum in this model is the sum $k_{J}=k_{1}+k_{2}$. We parametrize the momentum $k_{1}$ of the produced quark and the momentum $k_{2}$ of the produced antiquark as follows:

$$
\begin{aligned}
k_{1}= & \alpha_{1} p_{1}+\beta_{1} p_{2}+k_{1 \perp} \\
\equiv & x \alpha p_{1}+\frac{m^{2}-\left(\alpha k_{\perp}+l_{\perp}\right)^{2}}{x \alpha s} p_{2} \\
& +\alpha k_{\perp}+l_{\perp}, \quad k_{1}^{2}=m^{2}, \\
k_{2}= & \alpha_{2} p_{1}+\beta_{2} p_{2}+k_{2 \perp} \\
\equiv & x \bar{\alpha} p_{1}+\frac{m^{2}-\left(\bar{\alpha} k_{\perp}-l_{\perp}\right)^{2}}{x \bar{\alpha} s} p_{2} \\
& +\bar{\alpha} k_{\perp}-l_{\perp}, \quad k_{2}^{2}=m^{2}, \\
& M^{2}=\left(k_{1}+k_{2}\right)^{2} \equiv \frac{m^{2}-l_{\perp}^{2}}{\alpha \bar{\alpha}},
\end{aligned}
$$

with $\bar{\alpha}=1-\alpha$. Thus,

$$
\begin{aligned}
\delta(x- & {\left.\left[\alpha_{M}\right]\right) \delta^{2}\left(k_{\perp}-\left[p_{M \perp}\right]\right)\left[\frac{d^{2} p_{M}}{(2 \pi)^{3} 2 E_{M}}\right] } \\
= & \delta\left(x-\alpha_{1}-\alpha_{2}\right) \delta^{2}\left(k_{\perp}-k_{1 \perp}-k_{2 \perp}\right) \\
& \times \frac{d^{3} k_{1}}{(2 \pi)^{3} 2 E_{1}} \frac{d^{3} k_{2}}{(2 \pi)^{3} 2 E_{2}} \\
= & \delta\left(x-\alpha_{V}\right) \delta^{2}\left(k_{\perp}-k_{V \perp}\right) \frac{1}{4(2 \pi)^{6}} \frac{d \alpha d^{2} l_{\perp}}{\alpha \bar{\alpha}} d y_{V} d^{2} k_{V \perp},
\end{aligned}
$$

which leads, by taking into account (2.13), to the differential cross section in the color evaporation model having the form 


$$
\begin{aligned}
\frac{d \sigma}{d y_{V} d\left|p_{V \perp}\right| d \phi_{V} d y_{J} d\left|p_{J \perp}\right| d \phi_{J}}= & F_{J / \psi} \int d x g(x) d y H^{q}(d y) d^{2} k_{\perp} \int_{4 m^{2}}^{4 M_{D}^{2}} d M^{2} \delta\left(M^{2}-\frac{m^{2}-l_{\perp}^{2}}{\alpha \bar{\alpha}}\right) \\
& \times \frac{\left|p_{V \perp}\right| \sqrt{2} \delta\left(x-\alpha_{V}\right) \delta^{2}\left(k_{\perp}-p_{V \perp}\right)}{2^{5} \pi^{4} s^{2}\left(N^{2}-1\right)^{2} k_{\perp}^{2} x} \frac{d \alpha d^{2} l_{\perp}}{\alpha \bar{\alpha}} \sum_{\lambda_{k_{1}} \lambda_{k_{2}}} \mathcal{A}_{i \perp}^{a b} g_{\perp}^{i j}\left(\mathcal{A}_{j \perp}^{a b}\right)^{*} V_{q}^{(0)}\left(k_{\perp}, y\right),
\end{aligned}
$$

with $y_{V}=\ln \left(\alpha_{V} / \sqrt{\frac{M^{2}-p_{V \perp}^{2}}{s}}\right)$, from which we read off the $J / \psi$ production vertex in the color evaporation model:

$V_{J / \psi}^{(\mathrm{CEM})}\left(k_{\perp}, x\right)=F_{J / \psi} \int_{4 m^{2}}^{4 M_{D}^{2}} d M^{2} \delta\left(M^{2}-\frac{m^{2}-l_{\perp}^{2}}{\alpha \bar{\alpha}}\right) \frac{d \alpha d^{2} l_{\perp}}{\alpha \bar{\alpha}} \sum_{\lambda_{k_{1}} \lambda_{k_{2}}} \mathcal{A}_{i \perp}^{a b} g_{\perp}^{i j}\left(\mathcal{A}_{j \perp}^{a b}\right)^{*} \times \frac{\left|p_{V \perp}\right| \sqrt{2} \delta\left(x-\alpha_{V}\right) \delta^{2}\left(k_{\perp}-p_{V \perp}\right)}{2^{5} \pi^{4} s^{2}\left(N^{2}-1\right)^{2} k_{\perp}^{2} x}$.

The contribution to the hard part of the vertex in the Born approximation is given by three diagrams analogous to the ones of the color octet NRQCD contribution, except for the absence of any Fierz projection, since we simply deal with open quark-antiquark production. These diagrams are shown in Fig. 5. The hard part then reads

$$
\begin{aligned}
\mathcal{A}_{i \perp}^{a b}= & \bar{u}\left(k_{1}\right)\left[\left(-i g \gamma_{i \perp} t^{a}\right) \frac{i\left(-x \hat{p}_{1}+\hat{k}_{1}+m\right)}{\left(-x p_{1}+k_{1}\right)^{2}-m^{2}}\left(-i g t^{b} \hat{p}_{2}\right)\right. \\
& +\left(-i g t^{b} \hat{p}_{2}\right) \frac{i\left(x \hat{p}_{1}-\hat{k}_{2}+m\right)}{\left(x p_{1}-k_{2}\right)^{2}-m^{2}}\left(-i g \gamma_{i \perp} t^{a}\right) \\
& \left.+g f^{a b c}\left(-2 p_{2}^{\nu} k_{i \perp}+g_{i \perp}^{\nu} x s\right) \frac{(-i)}{M^{2}}\left(-i g \gamma_{\nu} t^{c}\right)\right] v\left(k_{2}\right) .
\end{aligned}
$$

Thus its contribution to the $J / \psi$ production vertex has the form

$$
\sum_{\lambda_{k_{1}} \lambda_{k_{2}}}\left(\mathcal{A}_{i \perp}^{a b}\right)^{*} g_{\perp}^{i j} \mathcal{A}_{j \perp}^{a b}=\frac{g^{4}}{4}\left(c_{a} \operatorname{Tr}_{a}+c_{b} \operatorname{Tr}_{b}\right)
$$

where the two color structures are given by

$$
\begin{aligned}
& c_{a}=\frac{f^{a b c} f^{a b c}}{2}=\frac{N\left(N^{2}-1\right)}{2}, \\
& c_{b}=\frac{\delta^{a b} \delta^{a b}}{N^{2}}+\frac{d^{a b c} d^{a b c}}{2}=\frac{N^{2}-1}{N^{2}}\left(1+\frac{N\left(N^{2}-4\right)}{2}\right),
\end{aligned}
$$

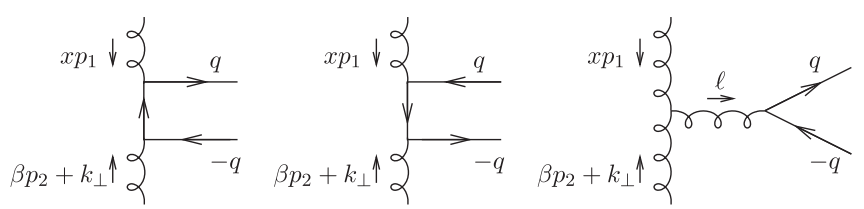

FIG. 5. The 3 diagrams contributing to the amplitude in the color evaporation model. and the two corresponding coefficients read

$$
\begin{aligned}
\operatorname{Tr}_{a}= & -4 s\left[\alpha_{2} \beta_{1}\left(-\frac{1}{\beta_{1}}+\frac{2 x s}{M^{2}}\right)^{2}+\alpha_{1} \beta_{2}\left(-\frac{1}{\beta_{2}}+\frac{2 x s}{M^{2}}\right)^{2}\right] \\
& -8 m^{2}\left(\frac{2 x s}{M^{2}}-\frac{1}{\beta_{2}}\right)\left(\frac{2 x s}{M^{2}}-\frac{1}{\beta_{1}}\right)+\frac{8}{x}\left[\frac{k_{1 \perp}}{\beta_{1}}+\frac{k_{2 \perp}}{\beta_{2}}-\frac{2 x s}{M^{2}} k_{\perp}\right] \\
& \cdot\left[\alpha_{2}\left(\left(\frac{\alpha_{1}}{x}-1\right) \frac{1}{\beta_{1}}+\frac{2 x s}{M^{2}}\right) k_{1 \perp}\right. \\
& \left.+\alpha_{1}\left(\left(\frac{\alpha_{2}}{x}-1\right) \frac{1}{\beta_{2}}+\frac{2 x s}{M^{2}}\right) k_{2 \perp}-\frac{2 \alpha_{1} \alpha_{2} s}{M^{2}} k_{\perp}\right] \\
= & -4 s x\left[\bar{\alpha} \beta_{1}\left(-\frac{1}{\beta_{1}}+\frac{2 x s}{M^{2}}\right)^{2}+\alpha \beta_{2}\left(-\frac{1}{\beta_{2}}+\frac{2 x s}{M^{2}}\right)^{2}\right] \\
& -8 m^{2}\left(\frac{2 x s}{M^{2}}-\frac{1}{\beta_{2}}\right)\left(\frac{2 x s}{M^{2}}-\frac{1}{\beta_{1}}\right)+8\left[\frac{k_{1 \perp}}{\beta_{1}}+\frac{k_{2 \perp}}{\beta_{2}}-\frac{2 x s}{M^{2}} k_{\perp}\right] \\
& \cdot\left[\bar{\alpha}\left(-\frac{\bar{\alpha}}{\beta_{1}}+\frac{2 x s}{M^{2}}\right) k_{1 \perp}+\alpha\left(-\frac{\alpha}{\beta_{2}}+\frac{2 x s}{M^{2}}\right) k_{2 \perp}-\alpha \bar{\alpha} \frac{2 x s}{M^{2}} k_{\perp}\right],
\end{aligned}
$$

and

$$
\begin{aligned}
\operatorname{Tr}_{b}= & -4 s\left(\frac{\alpha_{2}}{\beta_{1}}+\frac{\alpha_{1}}{\beta_{2}}\right) \\
& +8\left[\frac{\alpha_{2}}{x}\left(\frac{\alpha_{1}}{x}-1\right) \frac{k_{1 \perp}}{\beta_{1}}-\frac{\alpha_{1}}{x}\left(\frac{\alpha_{2}}{x}-1\right) \frac{k_{2 \perp}}{\beta_{2}}\right] \cdot\left[\frac{k_{1 \perp}}{\beta_{1}}-\frac{k_{2 \perp}}{\beta_{2}}\right] \\
& +\frac{8 m^{2}}{\beta_{1} \beta_{2}} \\
= & -4 s x\left(\frac{\bar{\alpha}}{\beta_{1}}+\frac{\alpha}{\beta_{2}}\right)-8\left[\bar{\alpha}^{2} \frac{k_{1 \perp}}{\beta_{1}}-\alpha^{2} \frac{k_{2 \perp}}{\beta_{2}}\right] \cdot\left[\frac{k_{1 \perp}}{\beta_{1}}-\frac{k_{2 \perp}}{\beta_{2}}\right] \\
& +\frac{8 m^{2}}{\beta_{1} \beta_{2}} .
\end{aligned}
$$

Using the fact that 


$$
\begin{aligned}
& k_{1 \perp} \underset{k_{\perp} \rightarrow 0}{\sim} l_{\perp}, \quad k_{2 \perp} \underset{k_{\perp} \rightarrow 0}{\sim}-l_{\perp}, \quad \beta_{1} \underset{k_{\perp} \rightarrow 0}{\sim} \frac{m^{2}-l_{\perp}^{2}}{x \alpha s}, \\
& \beta_{2} \underset{k_{\perp} \rightarrow 0}{\sim} \frac{m^{2}-l_{\perp}^{2}}{x \bar{\alpha} s},
\end{aligned}
$$

as well as the kinematical relation (2.50), one can easily check that, as expected, both $\operatorname{Tr}_{a}$ and $\operatorname{Tr}_{b}$ vanish in the limit $k_{\perp} \rightarrow 0$.

\section{RESULTS}

In this section we compare the cross sections and azimuthal correlations between the $J / \psi$ meson and the jet obtained with the color singlet, color octet and color evaporation hadronization mechanisms, for two different values of the center of mass energy: $\sqrt{s}=8 \mathrm{TeV}$ and $\sqrt{s}=13 \mathrm{TeV}$. We consider equal values of the transverse momenta of the $J / \psi$ and the jet, $\left|p_{V \perp}\right|=\left|p_{J \perp}\right|=p_{\perp}$, and four different kinematical configurations:

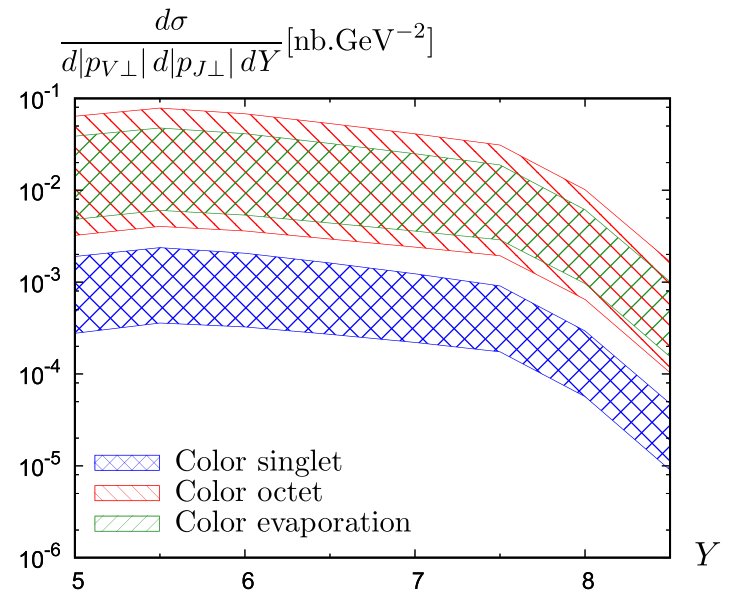

$$
0<y_{V}<2.5,-6.5<y_{J}<-5, p_{\perp}=10 \mathrm{GeV}
$$

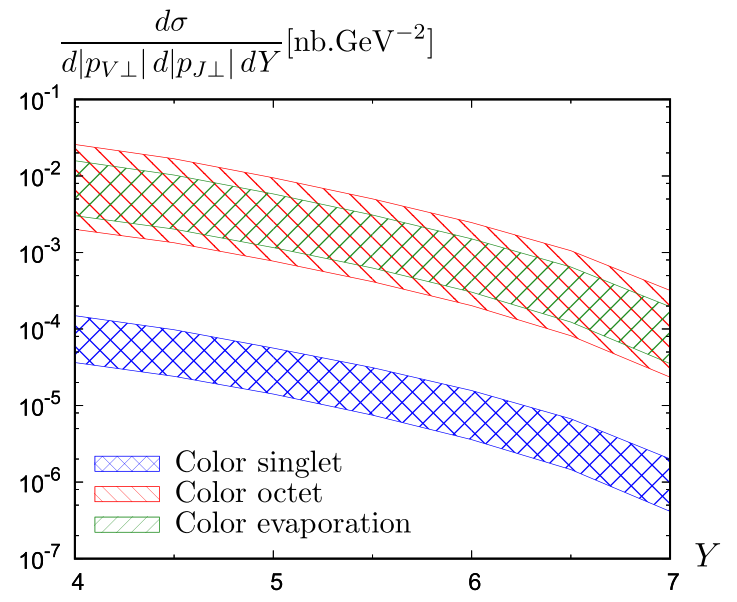

$0<y_{V}<2.5,-4.5<y_{J}<0, p_{\perp}=20 \mathrm{GeV}$ (i) $0<y_{V}<2.5,-6.5<y_{J}<5, p_{\perp}=10 \mathrm{GeV}$,

(ii) $0<y_{V}<2.5,-4.5<y_{J}<0, p_{\perp}=10 \mathrm{GeV}$,

(iii) $0<y_{V}<2.5,-4.5<y_{J}<0, p_{\perp}=20 \mathrm{GeV}$,

(iv) $0<y_{V}<2.5,-4.5<y_{J}<0, p_{\perp}=30 \mathrm{GeV}$.

The very backward jet in the first configuration could be measured for example with the CASTOR detector at CMS. An experimental study combining the CASTOR detector to tag the jet and the CMS tracking system to measure the $J / \psi$ meson would therefore allow to probe rapidity separations $Y \equiv y_{V}-y_{J}$ up to values as large as 9 . For the other three configurations we restrict the rapidity of the jet to $y_{J}>-4.5$ which corresponds to the typical values accessible by the main detectors at ATLAS and CMS. In this case the maximum rapidity separation is $Y=7$. Since a BFKL calculation is valid only for a large rapidity separation, we will only show results for $Y>4$. We use the BLM renormalization scale fixing procedure, see Ref. [15], which modifies the "natural" initial scale $\mu_{R, \text { init }}=\sqrt{\left|p_{V \perp}\right| \cdot\left|p_{J \perp}\right|}$ by

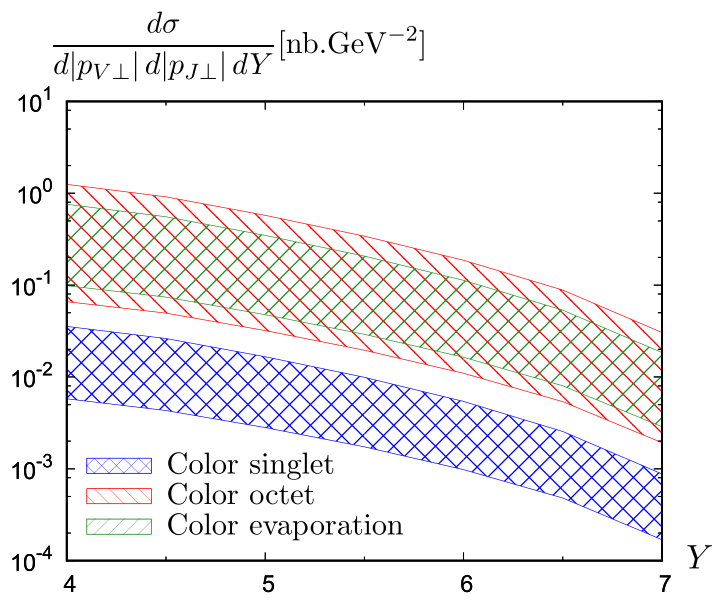

$$
0<y_{V}<2.5,-4.5<y_{J}<0, p_{\perp}=10 \mathrm{GeV}
$$

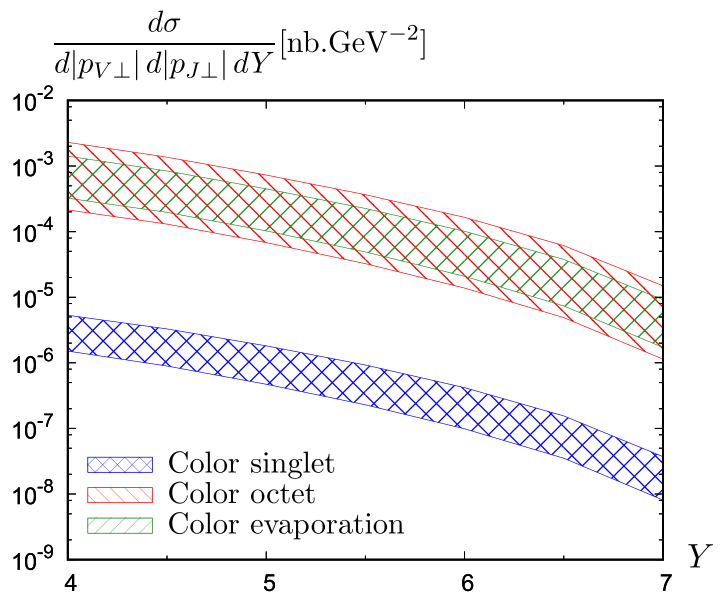

$0<y_{V}<2.5,-4.5<y_{J}<0, p_{\perp}=30 \mathrm{GeV}$

FIG. 6. Cross section at $\sqrt{s}=8 \mathrm{TeV}$ as a function of the relative rapidity $Y$ between the $J / \psi$ and the jet, in four different kinematical configurations. 

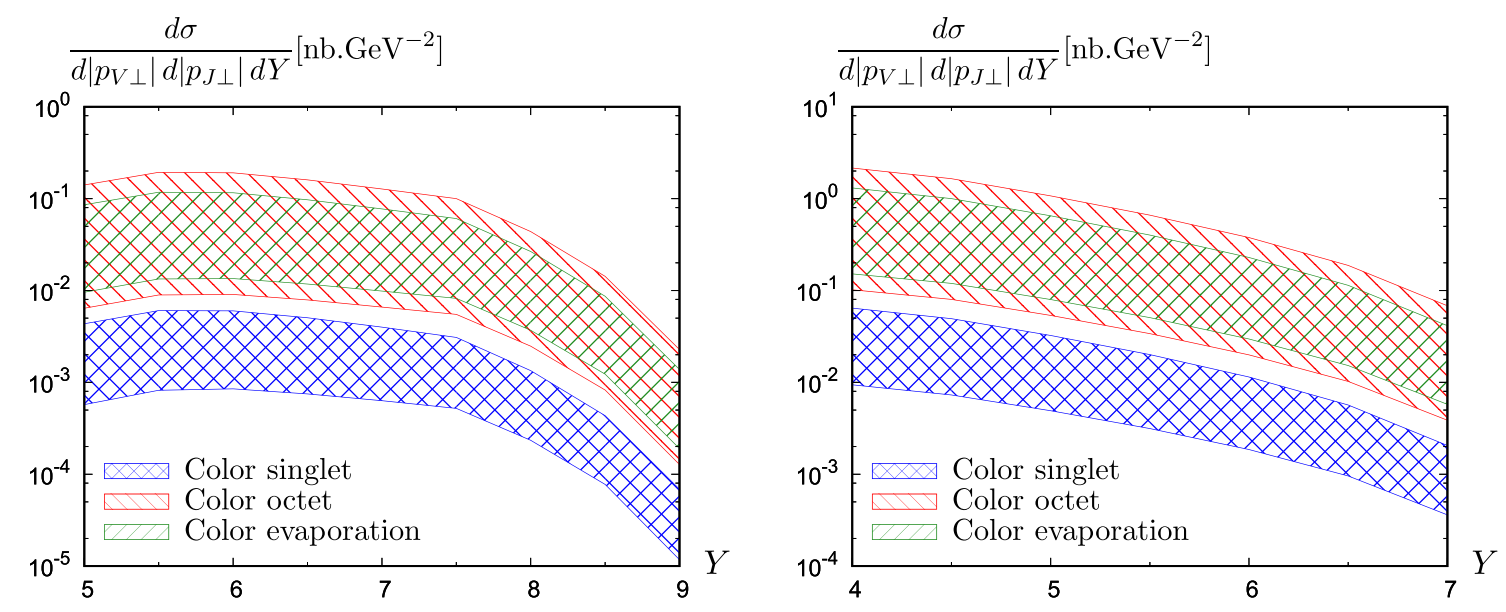

$$
0<y_{V}<2.5,-6.5<y_{J}<-5, p_{\perp}=10 \mathrm{GeV}
$$

$$
0<y_{V}<2.5,-4.5<y_{J}<0, p_{\perp}=10 \mathrm{GeV}
$$
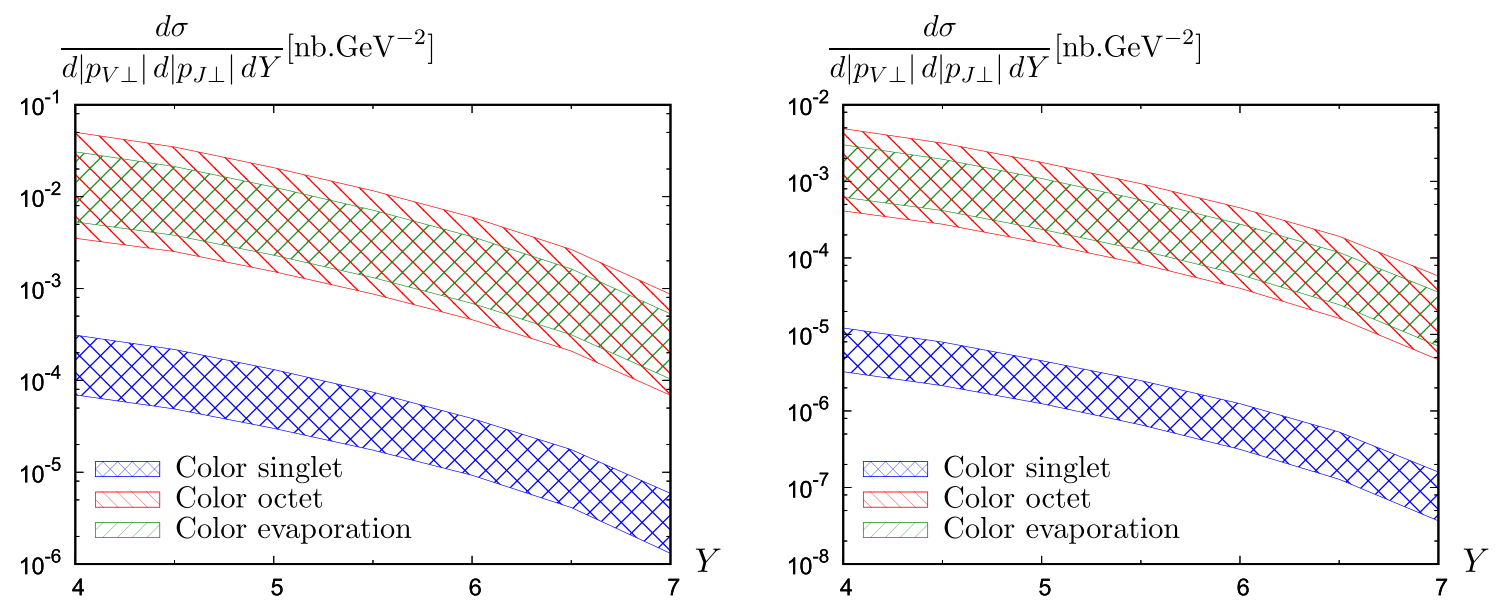

$$
0<y_{V}<2.5,-4.5<y_{J}<0, p_{\perp}=20 \mathrm{GeV}
$$

$0<y_{V}<2.5,-4.5<y_{J}<0, p_{\perp}=30 \mathrm{GeV}$

FIG. 7. Cross section at $\sqrt{s}=13 \mathrm{TeV}$ as a function of the relative rapidity $Y$ between the $J / \psi$ and the jet, in four different kinematical configurations.

$\mu_{R, \mathrm{BLM}}^{2}=\left|p_{V \perp}\right| \cdot\left|p_{J \perp}\right| \exp \left[\frac{1}{2} \chi_{0}(n, \gamma)-\frac{5}{3}+2\left(1+\frac{2}{3} I\right)\right]$,

where

$\chi_{0}(n, \gamma)=2 \psi(1)-\psi\left(\gamma+\frac{n}{2}\right)-\psi\left(1-\gamma+\frac{n}{2}\right)$

is the LL BFKL eigenvalue and $I=-2 \int_{0}^{1} d x \ln (x) /$ $\left[x^{2}-x+1\right] \simeq 2.3439$. The uncertainty band is computed in the same way as in Ref. [15] with the addition of the variation of the nonperturbative constants related to $\mathrm{J} / \psi$ hadronization in the ranges specified in the previous sections. We fix the charm quark mass to $m=1.5 \mathrm{GeV}$.

In Figs. 6 and 7 we show the differential cross section $\frac{d \sigma}{d\left|p_{V \perp}\right| d\left|p_{J \perp}\right| d Y}$ as a function of the rapidity separation $Y$ for the four kinematical cuts described above, for $\sqrt{s}=8 \mathrm{TeV}$ and $\sqrt{s}=13 \mathrm{TeV}$ respectively. We observe that in NRQCD the color octet contribution dominates over the color singlet one, especially at high $p_{\perp}$. The color evaporation model leads to similar results as the color octet NRQCD contribution. Note, however, that the absolute normalization of the cross section in the color evaporation model is not very well determined. As expected, the cross sections slightly increase when passing from $\sqrt{s}=8 \mathrm{TeV}$ to $\sqrt{s}=13 \mathrm{TeV}$, although this increase is much smaller than the uncertainties.

In Figs. 8 and 9 we show, in the same kinematics, the variation of $\langle\cos \varphi\rangle$ as a function of $Y$, where $\varphi$ is defined as $\varphi=\left|\phi_{V}-\phi_{J}-\pi\right|$, for $\sqrt{s}=8 \mathrm{TeV}$ and $\sqrt{s}=13 \mathrm{TeV}$ respectively. A value of $\varphi=0$ therefore corresponds to a back-to-back configuration for the $J / \psi$ and the jet and values of $\langle\cos \varphi\rangle$ close to unity are equivalent to a strong correlation. One can see from these figures that the values 

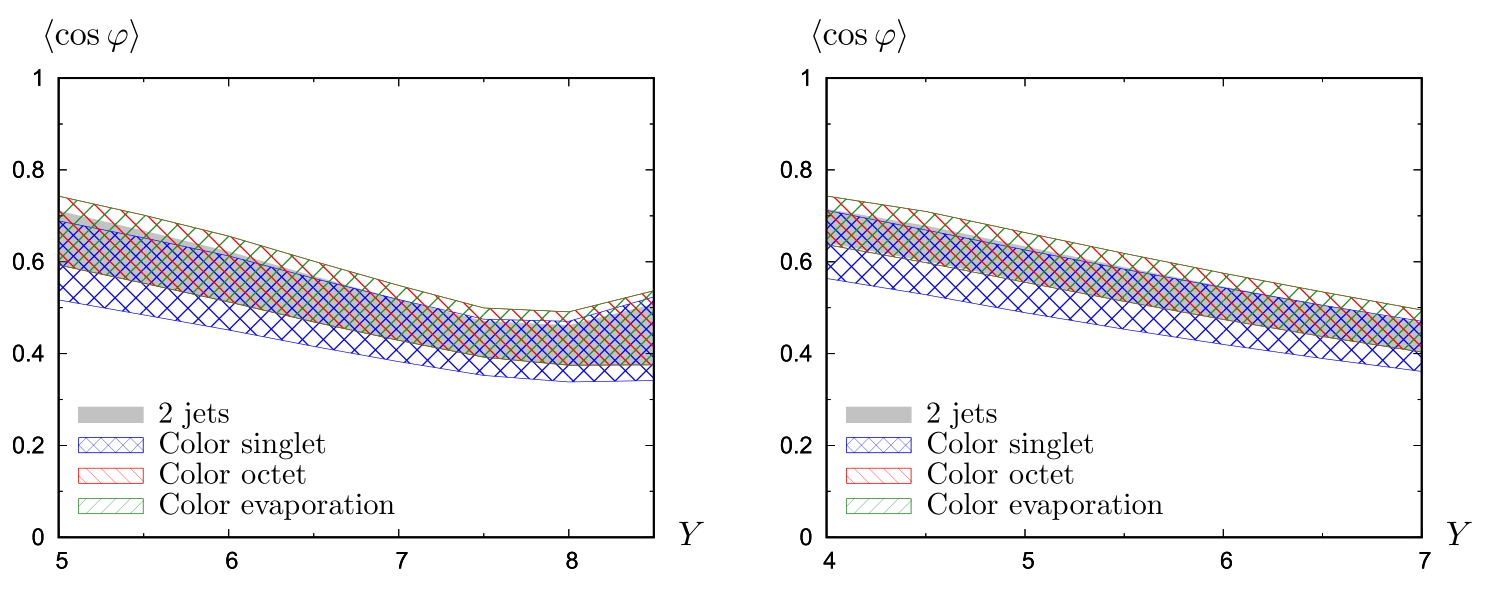

$0<y_{V}<2.5,-6.5<y_{J}<-5, p_{\perp}=10 \mathrm{GeV}$

$0<y_{V}<2.5,-4.5<y_{J}<0, p_{\perp}=10 \mathrm{GeV}$
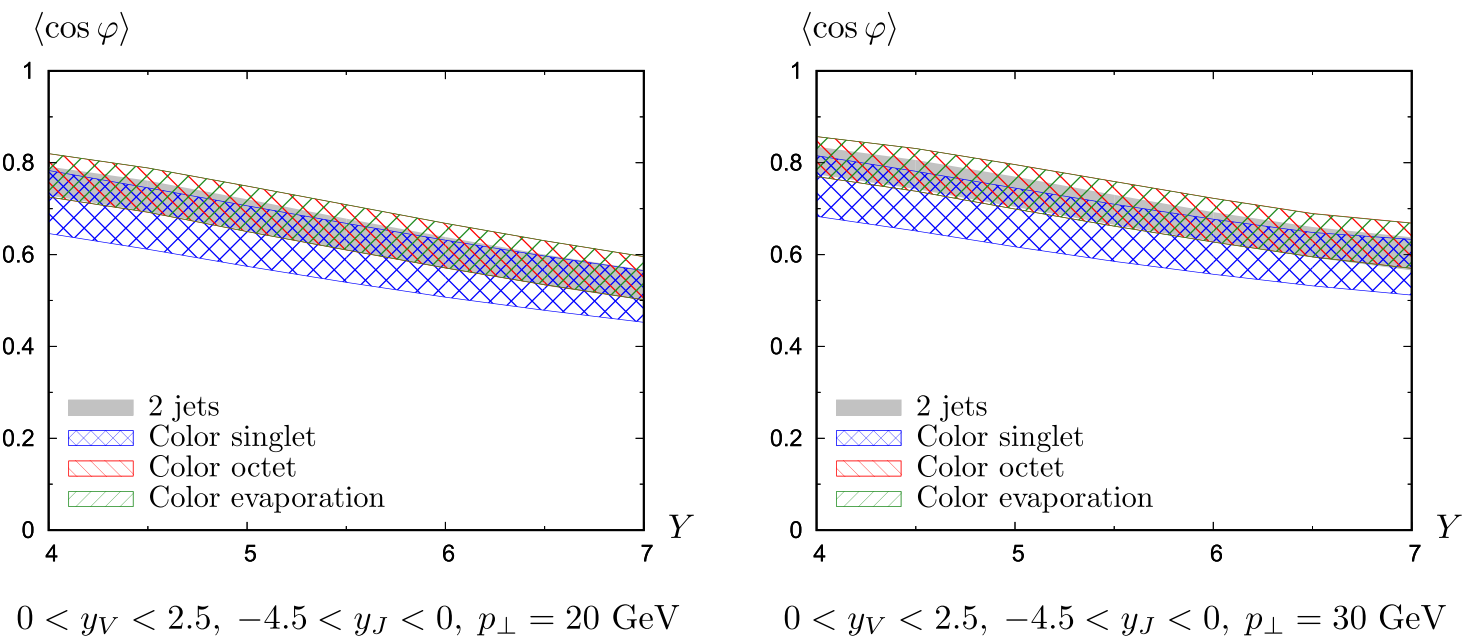

FIG. 8. Variation of $\langle\cos \varphi\rangle$ at $\sqrt{s}=8 \mathrm{TeV}$ as a function of the relative rapidity $Y$ between the $J / \psi$ and the jet, for the four kinematical cuts described in the text. The grey band corresponds to the results obtained when the $J / \psi$ production vertex is replaced by the leading order jet production vertex.

of $\langle\cos \varphi\rangle$ obtained with the three production mechanisms are compatible with each other as well as with the results obtained when the $J / \psi$ vertex is replaced by the leading order jet vertex shown for comparison. We note that passing from $\sqrt{s}=8 \mathrm{TeV}$ to $\sqrt{s}=13 \mathrm{TeV}$ increases very slightly the decorrelation effects.

One should note that these results could be significantly altered when taking into account the NLO corrections to the $J / \psi$ production vertex, as it is the case when passing from the LO to the NLO jet vertex, see Refs. $[13,14]$. The derivation of the NLO $J / \psi$ production vertex goes well beyond the scope of this work and is left for further studies.

\section{CONCLUSIONS}

The study of the present article was strongly motivated by the possibility of a first measurement by either ATLAS or CMS collaborations at the LHC going beyond the conventional Mueller-Navelet dijet production. Up to now Mueller-Navelet processes were considered as the inclusive production of two very forward jets separated by a large rapidity gap, and such processes are very promising ways of experimentally discriminating BFKL and pure collinear dynamics. Our study increases the number of phenomenologically important processes to study this discrimination, which is an outstanding issue in small-x physics.

We have shown that the study of the inclusive production of a forward $J / \psi$ and a very backward jet at the LHC leads to very promising cross sections, to be studied either at the ATLAS or CMS experiments. The possibility of tagging a high rapidity jet on one side, and a $J / \psi$ charmonium on the other side (although with a smaller absolute rapidity), can give access to BFKL resummation effects, since the relative rapidity up to roughly 7 (and even 9 for CASTOR) is theoretically just in the appropriate kinematical range. We have computed the required matrix elements, in the NRQCD color singlet and color octet approaches, as well 

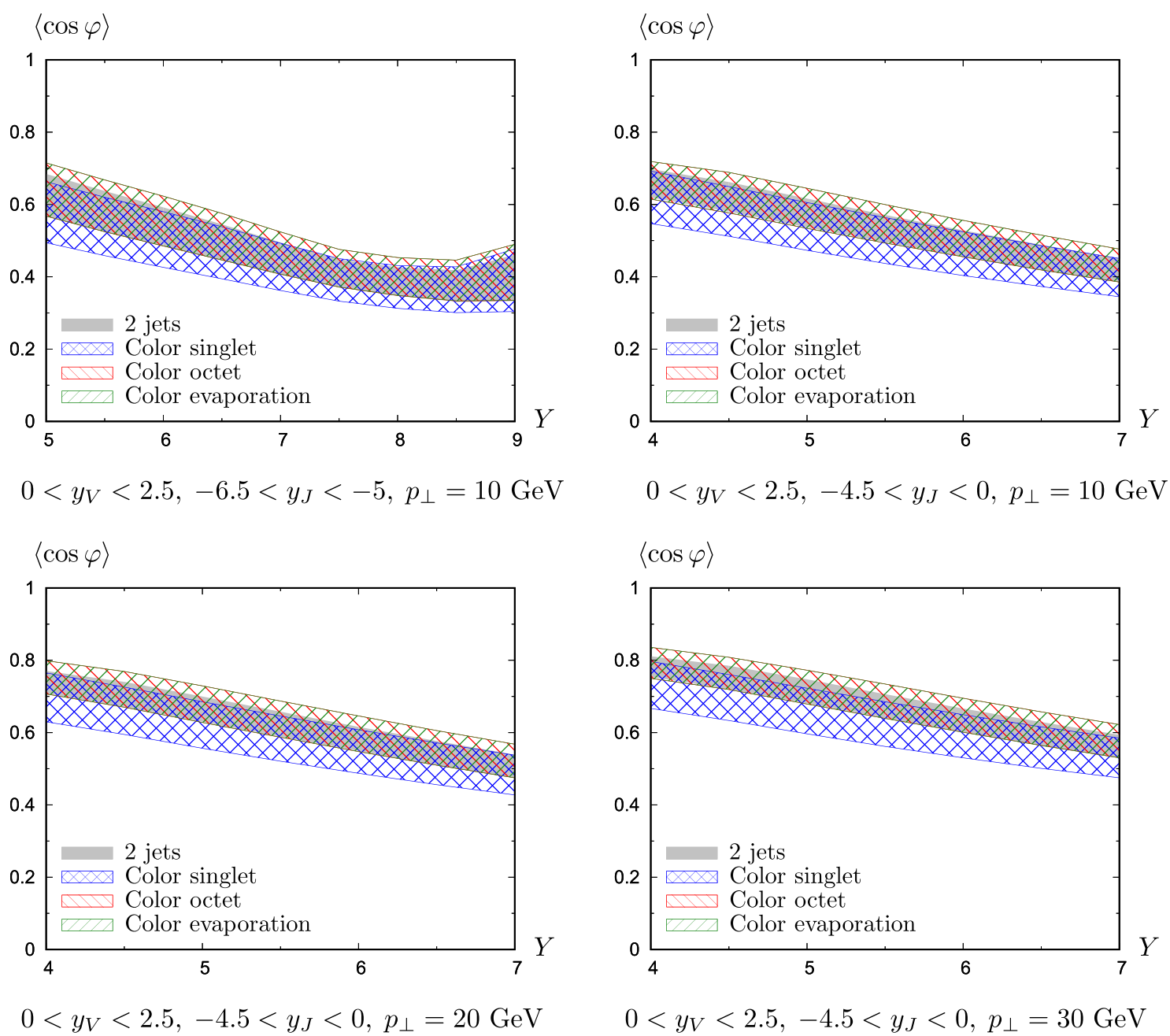

FIG. 9. Variation of $\langle\cos \varphi\rangle$ at $\sqrt{s}=13 \mathrm{TeV}$ as a function of the relative rapidity $Y$ between the $J / \psi$ and the jet, for the four kinematical cuts described in the text. The grey band corresponds to the results obtained when the $J / \psi$ production vertex is replaced by the leading order jet production vertex.

as in the color evaporation model. Our numerical results show that in the NRQCD approach, the color octet contribution dominates over the color singlet one, and the color evaporation model gives a prediction similar to the color octet NRQCD contribution. The study of the azimuthal correlations gives results which are very similar to the ones obtained in the Mueller-Navelet case (using for consistency one of the two jet vertices at LO, since the $J / \psi$ vertex is itself treated at $\mathrm{LO}$ ).

Our predictions for the rapidity distributions and azimuthal correlations of the meson and the jet shown in Figs. 6-9 include contributions from the nearly back-toback kinematics. One should note that in all three models considered in this article, Sudakov-type logarithms might appear analogously to the case of Mueller Navelet dijets in the almost back-to-back kinematics as discussed in Ref. [57]. The importance of Sudakov resummation effects for Mueller-Navelet jets is under investigation [58]. For the case of charmonium production considered here, the study of these Sudakov resummation effects, which goes beyond the scope of the present paper, could be performed in analogy with the works of Refs. [33,59].

The next stage, in order to get full NLL BFKL predictions for this process, would require to use the NLO expression for the charmonium production vertex, which has not yet been computed. This is left for future studies.

We did not include any double parton scattering contribution, which through two decorrelated BFKL ladders could lead to the same final state. In the case of MuellerNavelet jets, some of us have shown that this contribution is rather small with respect to the single BFKL ladder contribution [60], except potentially for large $s$ and small jet transverse momenta. For the present process, it would thus be interesting to study this contribution in the CASTOR kinematics.

Finally, a comparison between our predictions and analogous predictions purely based on collinear dynamics would be very interesting. This is left for future studies. 


\section{ACKNOWLEDGMENTS}

We thank Evgenii Baldin, Andrey Grabovsky, JeanPhilippe Lansberg and Hua-Sheng Shao for discussions. The research by R. B. and L.S. was supported by the National Science Center, Poland, Grant No. 2015/17/B/ ST2/01838. The research by B. D. was supported by the Academy of Finland, Project No. 273464, and by the
European Research Council, Grant No. ERC-2015CoG-681707. This work is partially supported by the French grant ANR PARTONS (Grant No. ANR-12MONU-0008-01), by the COPIN-IN2P3 agreement, by the Labex P2IO and by the Polish-French collaboration agreement Polonium. This work used computing resources from CSC-IT Center for Science in Espoo, Finland.
[1] V. S. Fadin, E. A. Kuraev, and L. N. Lipatov, On the Pomeranchuk Singularity in Asymptotically Free Theories, Phys. Lett. B60, 50 (1975).

[2] E. A. Kuraev, L. N. Lipatov, and V. S. Fadin, Multi-Reggeon processes in the Yang-Mills theory, Sov. Phys. JETP 44, 443 (1976).

[3] E. A. Kuraev, L. N. Lipatov, and V.S. Fadin, The Pomeranchuk singularity in nonabelian gauge theories, Sov. Phys. JETP 45, 199 (1977).

[4] I. I. Balitsky and L. N. Lipatov, The Pomeranchuk singularity in quantum chromodynamics, Sov. J. Nucl. Phys. 28, 822 (1978).

[5] H. Cheng and T. T. Wu, Photon-photon scattering close to the forward direction, Phys. Rev. D 1, 3414 (1970).

[6] G. Frolov and L. Lipatov, Sov. J. Nucl. Phys. 13, 333 (1971).

[7] V. Gribov, G. Frolov, and L. Lipatov, Yad. Fiz. 12, 994 (1970).

[8] S. Catani, M. Ciafaloni, and F. Hautmann, Gluon contributions to small $\mathrm{x}$ heavy flavor production, Phys. Lett. B 242, 97 (1990).

[9] S. Catani, M. Ciafaloni, and F. Hautmann, High-energy factorization and small x heavy flavor production, Nucl. Phys. B366, 135 (1991).

[10] J. C. Collins and R. K. Ellis, Heavy quark production in very high-energy hadron collisions, Nucl. Phys. B360, 3 (1991).

[11] E. M. Levin, M. G. Ryskin, Yu. M. Shabelski, and A. G. Shuvaev, Heavy quark production in semihard nucleon interactions, Sov. J. Nucl. Phys. 53, 657 (1991).

[12] A. H. Mueller and H. Navelet, An inclusive minijet crosssection and the bare Pomeron in QCD, Nucl. Phys. B282, 727 (1987).

[13] D. Colferai, F. Schwennsen, L. Szymanowski, and S. Wallon, Mueller Navelet jets at LHC-complete NLL BFKL calculation, J. High Energy Phys. 12 (2010) 026.

[14] B. Ducloué, L. Szymanowski, and S. Wallon, Confronting Mueller-Navelet jets in NLL BFKL with LHC experiments at $7 \mathrm{TeV}$, J. High Energy Phys. 05 (2013) 096.

[15] B. Ducloué, L. Szymanowski, and S. Wallon, Evidence for High-Energy Resummation Effects in Mueller-Navelet Jets at the LHC, Phys. Rev. Lett. 112, 082003 (2014).

[16] B. Ducloué, L. Szymanowski, and S. Wallon, Violation of energy-momentum conservation in Mueller-Navelet jets production, Phys. Lett. B 738, 311 (2014).
[17] F. Caporale, D. Yu. Ivanov, B. Murdaca, and A. Papa, Mueller-Navelet small-cone jets at LHC in next-to-leading BFKL, Nucl. Phys. B877, 73 (2013).

[18] F. Caporale, B. Murdaca, A. S. Vera, and C. Salas, Scale choice and collinear contributions to Mueller-Navelet jets at LHC energies, Nucl. Phys. B875, 134 (2013).

[19] F. Caporale, D. Yu. Ivanov, B. Murdaca, and A. Papa, Mueller-Navelet jets in next-to-leading order BFKL: Theory versus experiment, Eur. Phys. J. C 74, 3084 (2014); Erratum, Eur. Phys. J. C 75, 535(E) (2015).

[20] F. G. Celiberto, D. Yu. Ivanov, B. Murdaca, and A. Papa, Mueller-Navelet jets at LHC: BFKL versus high-energy DGLAP, Eur. Phys. J. C 75, 292 (2015).

[21] V. Khachatryan et al. (CMS Collaboration), Azimuthal decorrelation of jets widely separated in rapidity in $\mathrm{pp}$ collisions at $\sqrt{s}=7 \mathrm{TeV}$, J. High Energy Phys. 08 (2016) 139.

[22] N. Brambilla et al., Heavy quarkonium: progress, puzzles, and opportunities, Eur. Phys. J. C 71, 1534 (2011).

[23] G. T. Bodwin, E. Braaten, E. Eichten, S. L. Olsen, T. K. Pedlar, and J. Russ, Quarkonium at the Frontiers of High Energy Physics: A Snowmass White Paper, arXiv:1307.7425.

[24] A. Andronic et al., Heavy-flavour and quarkonium production in the LHC era: from proton-proton to heavy-ion collisions, Eur. Phys. J. C 76, 107 (2016).

[25] G. T. Bodwin, E. Braaten, and G. P. Lepage, Rigorous QCD analysis of inclusive annihilation and production of heavy quarkonium, Phys. Rev. D 51, 1125 (1995); Erratum, Phys. Rev. D 55, 5853(E) (1997).

[26] H. Fritzsch, Producing heavy quark flavors in hadronic collisions: A test of quantum chromodynamics, Phys. Lett. 67B, 217 (1977).

[27] F. Halzen, CVC for gluons and hadroproduction of quark flavors, Phys. Lett. 69B, 105 (1977).

[28] S. J. Brodsky, G. P. Lepage, and P. B. Mackenzie, On the elimination of scale ambiguities in perturbative quantum chromodynamics, Phys. Rev. D 28, 228 (1983).

[29] S. J. Brodsky, F. Hautmann, and D. E. Soper, Probing the QCD Pomeron in e+ e- Collisions, Phys. Rev. Lett. 78, 803 (1997); Erratum, Phys. Rev. Lett. 79, 3544(E) (1997).

[30] S. J. Brodsky, F. Hautmann, and D. E. Soper, Virtual photon scattering at high-energies as a probe of the short distance pomeron, Phys. Rev. D 56, 6957 (1997). 
[31] S. J. Brodsky, V. S. Fadin, V. T. Kim, L. N. Lipatov, and G. B. Pivovarov, The QCD pomeron with optimal renormalization, JETP Lett. 70, 155 (1999).

[32] S. J. Brodsky, V. S. Fadin, V. T. Kim, L. N. Lipatov, and G. B. Pivovarov, High-energy QCD asymptotics of photonphoton collisions, JETP Lett. 76, 249 (2002).

[33] J. W. Qiu, P. Sun, B. W. Xiao, and F. Yuan, Suppression of heavy quarkonium production in pA collisions at low transverse momentum, Phys. Rev. D 89, 034007 (2014).

[34] Z. B. Kang, Y. Q. Ma, and R. Venugopalan, Quarkonium production in high energy proton-nucleus collisions: CGC meets NRQCD, J. High Energy Phys. 01 (2014) 056.

[35] J. Bartels, D. Colferai, and G. P. Vacca, The NLO jet vertex for Mueller-Navelet and forward jets: The quark part, Eur. Phys. J. C 24, 83 (2002).

[36] J. Bartels, D. Colferai, and G. P. Vacca, The NLO jet vertex for Mueller-Navelet and forward jets: The gluon part, Eur. Phys. J. C 29, 235 (2003).

[37] V.S. Fadin and L. N. Lipatov, BFKL pomeron in the next-to-leading approximation, Phys. Lett. B 429, 127 (1998).

[38] M. Ciafaloni and G. Camici, Energy scale(s) and next-toleading BFKL equation, Phys. Lett. B 430, 349 (1998).

[39] B. Guberina, J. H. Kuhn, R. D. Peccei, and R. Ruckl, Rare decays of the $Z^{0}$, Nucl. Phys. B174, 317 (1980).

[40] R. Baier and R. Ruckl, Hadronic collisions: A quarkonium factory, Z. Phys. C 19, 251 (1983).

[41] R. Barbieri, R. Gatto, R. Kogerler, and Z. Kunszt, Meson hyperfine splittings and leptonic decays, Phys. Lett. 57B, 455 (1975).

[42] A. Billoire and A. Morel, Has asymptotic freedom something to do with charmonium?, Nucl. Phys. B135, 131 (1978).

[43] W. Celmaster, Lepton width suppression in vector mesons, Phys. Rev. D 19, 1517 (1979).

[44] C. Patrignani et al. (Particle Data Group Collaboration), Review of particle physics, Chin. Phys. C 40, 100001 (2016).

[45] R. Bain, L. Dai, A. Leibovich, Y. Makris, and T. Mehen, NRQCD Confronts LHCb Data on Quarkonium Production within Jets, Phys. Rev. Lett. 119, 032002 (2017).
[46] M. Butenschoen and B. A. Kniehl, World data of $J / \psi$ production consolidate NRQCD factorization at NLO, Phys. Rev. D 84, 051501 (2011).

[47] K.-T. Chao, Y.-Q. Ma, H.-S. Shao, K. Wang, and Y.-J. Zhang, $J / \psi$ Polarization at Hadron Colliders in Nonrelativistic QCD, Phys. Rev. Lett. 108, 242004 (2012).

[48] G. T. Bodwin, H. S. Chung, U.-R. Kim, and J. Lee, Fragmentation Contributions to $J / \psi$ Production at the Tevatron and the LHC, Phys. Rev. Lett. 113, 022001 (2014).

[49] E. J. Eichten and C. Quigg, Quarkonium wave functions at the origin, Phys. Rev. D 52, 1726 (1995).

[50] G. T. Bodwin, H. S. Chung, D. Kang, J. Lee, and C. Yu, Improved determination of color-singlet nonrelativistic QCD matrix elements for S-wave charmonium, Phys. Rev. D 77, 094017 (2008).

[51] P. L. Cho and A. K. Leibovich, Color octet quarkonia production, Phys. Rev. D 53, 150 (1996).

[52] P. L. Cho and A. K. Leibovich, Color octet quarkonia production. 2., Phys. Rev. D 53, 6203 (1996).

[53] M. Kramer, Quarkonium production at high-energy colliders, Prog. Part. Nucl. Phys. 47, 141 (2001).

[54] P. Hagler, R. Kirschner, A. Schafer, L. Szymanowski, and O. V. Teryaev, Direct $J / \psi$ hadroproduction in $k_{\perp}$ factorization and the color octet mechanism, Phys. Rev. D 63, 077501 (2001).

[55] R. E. Nelson, R. Vogt, and A. D. Frawley, Narrowing the uncertainty on the total charm cross section and its effect on the $J / \psi$ cross section, Phys. Rev. C 87, 014908 (2013).

[56] J. P. Lansberg and H. S. Shao, Associated production of a quarkonium and a $\mathrm{Z}$ boson at one loop in a quark-hadronduality approach, J. High Energy Phys. 10 (2016) 153.

[57] A. H. Mueller, L. Szymanowski, S. Wallon, B. W. Xiao, and F. Yuan, Sudakov resummations in Mueller-Navelet dijet production, J. High Energy Phys. 03 (2016) 096.

[58] B. Ducloué, A. H. Mueller, L. Szymanowski, S. Wallon, B. W. Xiao, and F. Yuan (to be published).

[59] E. L. Berger, J. w. Qiu, and Y. 1. Wang, Transverse momentum distribution of $v$ production in hadronic collisions, Phys. Rev. D 71, 034007 (2005).

[60] B. Ducloué, L. Szymanowski, and S. Wallon, Evaluating the double parton scattering contribution to Mueller-Navelet jets production at the LHC, Phys. Rev. D 92, 076002 (2015). 